\title{
Travelling Waves of a Diffusive Epidemic Model with Latency and Relapse
}

\author{
Zhiping Wang and Rui Xu \\ Institute of Applied Mathematics, Shijiazhuang Mechanical Engineering College, Shijiazhuang 050003, China \\ Correspondence should be addressed to Zhiping Wang; wzpwyu@163.com
}

Received 21 December 2012; Accepted 25 February 2013

Academic Editor: Zhen Jin

Copyright (c) 2013 Z. Wang and R. Xu. This is an open access article distributed under the Creative Commons Attribution License, which permits unrestricted use, distribution, and reproduction in any medium, provided the original work is properly cited.

An SEIR epidemic model with relapse and spatial diffusion is studied. By analyzing the corresponding characteristic equations, the local stability of each of the feasible steady states to this model is discussed. The existence of a travelling wave solution is established by using the technique of upper and lower solutions and Schauder's fixed point theorem. Numerical simulations are carried out to illustrate the main results.

\section{Introduction}

In most epidemic models, individuals are often divided into several classes such as susceptible, infective, and recovered classes. Some diseases such as human or bovine tuberculosis have a latency (see, e.g., $[1,2]$ and references therein): infected individuals do not infect other susceptible individuals until sometime later. Hence, it is reasonable to include a latent class in such disease models. Also, for some diseases, recovered individuals may relapse with reactivation of latent infection and revert back to the infective class. This recurrence of disease is an important feature of some animal and human diseases. For human tuberculosis, incomplete treatment can lead to relapse, but relapse can also occur in patients who took a full course of treatment and were declared cured [3-5]. Most tuberculosis in human adults (caused by Mycobacterium tuberculosis) in the USA results from reactivation of latent infection [3].

In order for a model to be more realistic, taking into consideration a general exposed distribution and the possibility of relapse, van den Driessche et al. [6] formulated and studied the following model:

$$
\begin{gathered}
\frac{\mathrm{d} S(t)}{\mathrm{d} t}=\mu N-\beta S(t) \frac{I(t)}{N}-\mu S(t), \\
E(t)=\int_{0}^{t} \beta S(\xi) \frac{I(\xi)}{N} e^{-\mu(t-\xi)} P(t-\xi) \mathrm{d} \xi,
\end{gathered}
$$

$$
\begin{aligned}
& \frac{\mathrm{d} R(t)}{\mathrm{d} t}=\gamma I(t)-(\mu+\delta) R(t), \\
& I(t)=N-S(t)-E(t)-R(t),
\end{aligned}
$$

where $N$ is the size of the population; $S, E, I$, and $R$ are the population sizes of susceptible, latent, infective, and recovered classes, respectively; $\delta>0$ is a constant rate at which an individual in the recovered class reverts to the infective class; $\mu>0$ is the recruitment rate and the death rate (this guarantees that $N$ can be assumed as a constant); $\beta>0$ is the transmission coefficient; $\gamma \geqslant 0$ is the recovery rate; $P(t)$ denotes the probability that a latent individual still remains in the latent class $t$ time units after entering the latent class.

As in [6], when $P(t)$ is a step function, that is,

$$
P(t)= \begin{cases}1, & 0<t<\tau \\ 0, & t>\tau\end{cases}
$$

by rescaling

$$
\begin{array}{ll}
\frac{S(t)}{N} \longrightarrow S(t), & \frac{E(t)}{N} \longrightarrow E(t), \\
\frac{I(t)}{N} \longrightarrow I(t), & \frac{R(t)}{N} \longrightarrow R(t),
\end{array}
$$


model (1) reduces to the following delay differential equation system:

$$
\begin{gathered}
S^{\prime}(t)=\mu-\mu S(t)-\beta S(t) I(t), \\
I^{\prime}(t)=\beta e^{-\mu \tau} S(t-\tau) I(t-\tau)+\delta R(t)-(\mu+\gamma) I(t), \\
R^{\prime}(t)=\gamma I(t)-(\mu+\delta) R(t),
\end{gathered}
$$

where $\tau>0$ can be explained as the average latent period of the disease. The global stability of the steady states for system (1) and (4) was analyzed in [6, 7].

Note that it is implicitly assumed that the population is well mixed, and the spatial mobility of individuals has been ignored in model (1) as well as most other epidemic models. In reality, the environment in which an individual lives is often heterogeneous making it necessary to distinguish the locations, and due to the large mobility of individuals within an area or even worldwide, spatially uniform models are not sufficient to give a realistic picture of a disease's transmission. For this reason, the spatial effects cannot be neglected in studying the spread of epidemics. If the environment is spatially continuous, random diffusion is often used to describe the mobility of the population, leading to models in the form of reaction-diffusion equations (see, e.g., [8-10]). Noble [9] applied reaction-diffusion theory to describe the spread of plague through Europe in the mid-14th century. Maidana and Yang [10] proposed a spatial model to analyze the West Nile Virus propagation across the USA, from east to west.

Motivated by the work mentioned above, the main purpose of this paper is to study the effect of the spatial diffusion of the individuals on the dynamics of an epidemic model with latency and relapse. If we further consider the spatial diffusion, then the epidemic model corresponding to (4) should be described by the following delayed reaction diffusion system:

$$
\begin{aligned}
\frac{\partial S(x, t)}{\partial t}= & d_{1} \frac{\partial^{2} S(x, t)}{\partial x^{2}}+\mu-\mu S(x, t)-\beta S(x, t) I(x, t), \\
\frac{\partial I(x, t)}{\partial t}= & d_{2} \frac{\partial^{2} I(x, t)}{\partial x^{2}}+\beta e^{-\mu \tau} S(x, t-\tau) I(x, t-\tau) \\
& +\delta R(x, t)-(\mu+\gamma) I(x, t), \\
\frac{\partial R(x, t)}{\partial t}= & d_{3} \frac{\partial^{2} R(x, t)}{\partial x^{2}}+\gamma I(x, t)-(\mu+\delta) R(x, t),
\end{aligned}
$$

with initial conditions

$$
\begin{array}{r}
S(x, t)=\rho_{1}(x, t), \quad I(x, t)=\rho_{2}(x, t), \quad R(x, t)=\rho_{3}(x, t), \\
t \in[-\tau, 0], x \in \bar{\Omega} .
\end{array}
$$

In problem (5) and (6), $d_{1}, d_{2}$, and $d_{3}$ are the spatial diffusion rates of susceptible, infective, and recovered individuals, respectively. The functions $\rho_{i}(x, t) \in \mathscr{C}=C([-\tau, 0], X)(i=$ $1,2,3)$ and $X$ is defined by

$$
\begin{aligned}
X=\left\{S, I, R \in W^{2,2}(\Omega): \frac{\partial S(x, t)}{\partial n}\right. & \\
= & \left.\frac{\partial I(x, t)}{\partial n}=\frac{\partial R(x, t)}{\partial n}=0, x \in \partial \Omega\right\},
\end{aligned}
$$

with the inner product $\langle\cdot, \cdot\rangle$, where $\partial / \partial n$ denotes the outward normal derivative on $\partial \Omega$.

The organization of this paper is as follows. In the next section, by analyzing the corresponding characteristic equations, the local stability of an endemic steady state and a disease-free steady state of system (5) is discussed. In Section 3, by constructing a pair of upper-lower solutions, we use the cross-iteration method and the Schauder's fixed point theorem to prove the existence of travelling wave solutions to system (5). In Section 4, numerical simulations are carried out to illustrate the main theoretical results, and by combining with numeric simulations, we discuss the effects of some parameters on the dynamics of system (5). A brief conclusion is given in the last section.

\section{Local Stability}

In this section, by analyzing the corresponding characteristic equations, we investigate the local stability of steady states to system (5) with the initial conditions (6) and the homogeneous Neumann boundary conditions

$$
\frac{\partial S(x, t)}{\partial n}=\frac{\partial I(x, t)}{\partial n}=\frac{\partial R(x, t)}{\partial n}=0, \quad t \geqslant 0, x \in \partial \Omega,
$$

respectively. The homogeneous Neumann boundary conditions imply that the populations do not move across the boundary $\partial \Omega$.

Let

$$
\mathscr{R}_{0}=\frac{\beta e^{-\mu \tau}(\mu+\delta)}{\mu(\mu+\delta+\gamma)} .
$$

$\mathscr{R}_{0}$ is called the basic reproductive number of system (5), which describes the average number of newly infected individuals at the beginning of the infectious process. It is easy to show that system (5) always has a disease-free uniform steady state $E_{0}(1,0,0)$. Further, if $\mathscr{R}_{0}>1$, system (5) has a unique endemic steady state $E^{*}\left(S^{*}, I^{*}, R^{*}\right)$, where

$$
S^{*}=\frac{\mu(\mu+\delta+\gamma) e^{\mu \tau}}{\beta(\mu+\delta)},
$$

$$
\begin{gathered}
I^{*}=\frac{\beta e^{-\mu \tau}(\mu+\delta)-\mu(\mu+\delta+\gamma)}{\beta(\mu+\delta+\gamma)}, \\
R^{*}=\frac{\gamma}{\mu+\delta} I^{*} .
\end{gathered}
$$

Let $\widehat{E}\left(S^{0}, I^{0}, R^{0}\right)$ represent any feasible uniform steady state of system (5). If we set $\bar{S}=S-S^{0}, \bar{I}=I-I^{0}, \bar{R}=R-R^{0}$, 
and drop the bars for simplicity of notations, then (5) can be transformed into the following equivalent system:

$$
\begin{aligned}
& \frac{\partial S(x, t)}{\partial t} \\
& \quad=d_{1} \frac{\partial^{2} S(x, t)}{\partial x^{2}}-\mu S(x, t)-\beta S(x, t)\left(I(x, t)+I^{0}\right) \\
& \quad-\beta S^{0} I(x, t), \\
& \frac{\partial I(x, t)}{\partial t} \\
& \quad+d_{2} \frac{\partial^{2} I(x, t)}{\partial x^{2}} \\
& \quad+\beta e^{-\mu \tau}\left[S(x, t-\tau)\left(I(x, t-\tau)+I^{0}\right)+S^{0} I(x, t-\tau)\right] \\
& \quad+\delta R(x, t)-(\mu+\gamma) I(x, t), \\
& \frac{\partial R(x, t)}{\partial t} \\
& \quad d_{3} \frac{\partial^{2} R(x, t)}{\partial x^{2}}+\gamma I(x, t)-(\mu+\delta) R(x, t) .
\end{aligned}
$$

Denote $U=(S(\cdot, t), I(\cdot, t), R(\cdot, t))^{T}$. Then the linearization of (11) can be rewritten as an abstract differential equation in the phase space $\mathscr{C}=C([-\tau, 0], X)$,

$$
\dot{U}(t)=D \Delta U(t)+L\left(U_{t}\right),
$$

where $D=\operatorname{diag}\left(d_{1}, d_{2}, d_{3}\right)$, and $L$ is defined by

$$
\begin{aligned}
L(\phi)= & \left(\begin{array}{ccc}
-\mu-\beta I^{0} & -\beta S^{0} & 0 \\
0 & -(\mu+\gamma) & \delta \\
0 & \gamma & -(\mu+\delta)
\end{array}\right)\left(\begin{array}{l}
\phi_{1}(0) \\
\phi_{2}(0) \\
\phi_{3}(0)
\end{array}\right) \\
& +\left(\begin{array}{ccc}
0 & & 0 \\
\beta e^{-\mu \tau} I^{0} & \beta e^{-\mu \tau} S^{0} & 0 \\
0 & 0 & 0
\end{array}\right)\left(\begin{array}{l}
\phi_{1}(-\tau) \\
\phi_{2}(-\tau) \\
\phi_{3}(-\tau)
\end{array}\right)
\end{aligned}
$$

for $\phi=\left(\phi_{1}, \phi_{2}, \phi_{3}\right)^{T} \in \mathscr{C}$, and its characteristic equation is

$$
\lambda y-D \Delta y-L\left(e^{\lambda} y\right)=0
$$

where $y \in \operatorname{dom}(\Delta)$ and $y \neq 0, \operatorname{dom}(\Delta) \subset X$.

From the properties of the Laplacian operator defined on the bounded domain, the operator $\Delta$ on $X$ has the eigenvalues $-k^{2}$ with the relative eigenfunctions

$$
\beta_{k}^{1}=\left(\gamma_{k}, 0,0\right)^{T}, \quad \beta_{k}^{2}=\left(0, \gamma_{k}, 0\right)^{T}, \quad \beta_{k}^{3}=\left(0,0, \gamma_{k}\right)^{T},
$$

where $\gamma_{k}=\cos (k x), k \in N_{0}=\{0,1,2, \ldots\}$. Clearly, $\left(\beta_{k}^{1}, \beta_{k}^{2}\right.$, $\left.\beta_{k}^{3}\right)_{k=0}^{\infty}$ construct a basis of the phase space $X$, and therefore, any element $y$ in $X$ can be expanded as Fourier series in the following form:

$$
\begin{gathered}
y=\sum_{k=0}^{\infty} Y_{K}^{T}\left(\beta_{k}^{1}, \beta_{k}^{2}, \beta_{k}^{3}\right)^{T}, \\
Y_{K}^{T}=\left(\left\langle y, \beta_{k}^{1}\right\rangle,\left\langle y, \beta_{k}^{2}\right\rangle,\left\langle y, \beta_{k}^{3}\right\rangle\right) .
\end{gathered}
$$

Some simple computations show that

$$
L\left(\phi^{T}\left(\beta_{k}^{1}, \beta_{k}^{2}, \beta_{k}^{3}\right)^{T}\right)=L\left(\phi^{T}\right)\left(\beta_{k}^{1}, \beta_{k}^{2}, \beta_{k}^{3}\right)^{T}, \quad k \in N_{0} .
$$

From (16) and (17), (14) is equivalent to

$$
\sum_{k=0}^{\infty} Y_{K}^{T}\left(\lambda I_{2}+D k^{2}-M\right)\left(\beta_{k}^{1}, \beta_{k}^{2}, \beta_{k}^{3}\right)^{T}=0
$$

where

$$
M=\left(\begin{array}{ccc}
-\mu-\beta I^{0} & -\beta S^{0} & 0 \\
\beta e^{-(\mu+\lambda) \tau} I^{0} & \beta e^{-(\mu+\lambda) \tau} S^{0}-(\mu+\gamma) & \delta \\
0 & \gamma & -(\mu+\delta)
\end{array}\right) .
$$

If $E^{0}=E_{0}$, (18) is equivalent to

$$
\begin{aligned}
& \left(\lambda+k^{2} d_{1}+\mu\right) \\
& \times\left[\left(\lambda+k^{2} d_{2}+\mu+\gamma\right)\left(\lambda+k^{2} d_{3}+\mu+\delta\right)-\delta \gamma\right. \\
& \left.\quad-\left(\lambda+k^{2} d_{3}+\mu+\delta\right) \beta e^{-\mu \tau} e^{-\lambda \tau}\right]=0 .
\end{aligned}
$$

Clearly, for any $i \geqslant 1,(20)$ has a negative root $-k^{2} d_{1}-\mu$. Its other roots satisfy

$$
\begin{aligned}
g_{1}(\lambda) \triangleq & \left(\lambda+k^{2} d_{2}+\mu+\gamma\right)\left(\lambda+k^{2} d_{3}+\mu+\delta\right) \\
& -\delta \gamma-\left(\lambda+k^{2} d_{3}+\mu+\delta\right) \beta e^{-\mu \tau} e^{-\lambda \tau}=0
\end{aligned}
$$

If $\mathscr{R}_{0}>1$, then for $i=1$, we get that, for $\lambda$ real,

$$
\begin{gathered}
g_{1}(0)=\mu(\mu+\delta+\gamma)-(\mu+\delta) \beta e^{-\mu \tau}<0, \\
\lim _{\lambda \rightarrow+\infty} g_{1}(\lambda)=\infty .
\end{gathered}
$$

Hence, when $i=1$, (21) has a positive real root, and accordingly, $E_{0}$ is unstable.

If $\mathscr{R}_{0}<1$ and $\tau=0$, we have

$$
\begin{aligned}
g_{1}(\lambda)= & \lambda^{2}+\left(k^{2} d_{2}+k^{2} d_{3}+2 \mu+\gamma+\delta-\beta\right) \lambda \\
& +\left(k^{2} d_{2}+\mu+\gamma-\beta\right)\left(k^{2} d_{3}+\mu+\delta\right)-\delta \gamma>0,
\end{aligned}
$$

which means that the roots of (21) are negative constants, then $E_{0}$ is locally asymptotically stable. For $\tau>0$, if $i \sigma(\sigma>0)$ 
is a solution of (21), separating the real and imaginary parts, we derive that

$$
\begin{gathered}
-\sigma^{2}+A_{0}=A_{1} \cos \sigma \tau+A_{2} \sigma \sin \sigma \tau, \\
A_{3} \sigma=A_{2} \sigma \cos \sigma \tau-A_{1} \sin \sigma \tau,
\end{gathered}
$$

where

$$
\begin{gathered}
A_{0}=\left(k^{2} d_{2}+\mu+\gamma\right)\left(k^{2} d_{3}+\mu+\delta\right)-\delta \gamma, \\
A_{1}=\left(k^{2} d_{3}+\mu+\delta\right) \beta e^{-\mu \tau}, \\
A_{2}=\beta e^{-\mu \tau}, \quad A_{3}=k^{2}\left(d_{2}+d_{3}\right)+2 \mu+\gamma+\delta .
\end{gathered}
$$

Squaring and adding the two equations of (24), it follows that

$$
\sigma^{4}+\left(A_{3}^{2}-2 A_{0}-A_{2}^{2}\right) \sigma^{2}+A_{0}^{2}-A_{1}^{2}=0 .
$$

Note that if $R_{0}<1$, we obtain

$$
\begin{aligned}
A_{3}^{2}-2 A_{0}-A_{2}^{2}= & \left(k^{2} d_{2}+\mu+\gamma\right)^{2}+\left(k^{2} d_{3}+\mu+\gamma\right)^{2} \\
& +2 \delta \gamma-\beta^{2} e^{-2 \mu \tau} \\
\geqslant & (\mu+\gamma)^{2}+(\mu+\delta)^{2}+2 \delta \gamma-\beta^{2} e^{-2 \mu \tau} \\
\geqslant & \mu^{2}>0, \\
A_{0}-A_{1} \geqslant & (\mu+\delta)\left(\mu+\gamma-\beta e^{-\mu \tau}\right)-\delta \gamma \\
= & \mu(\mu+\delta+\gamma)-\beta(\mu+\delta) e^{-\mu \tau}>0 .
\end{aligned}
$$

Hence, (26) has no positive roots for all $i \geqslant 1$. Therefore, if $\mathscr{R}_{0}<1, E_{0}$ is locally asymptotically stable for all $\tau>0$.

If $E^{0}=E^{*}$, (18) is equivalent to

$$
\lambda^{3}+p_{2} \lambda^{2}+p_{1} \lambda+p_{0}+\left(q_{2} \lambda^{2}+q_{1} \lambda+q_{0}\right) e^{-\lambda \tau}=0
$$

where

$$
\begin{aligned}
p_{0}= & \left(k^{2} d_{1}+\mu+\beta I^{*}\right)\left[\left(k^{2} d_{2}+\mu+\gamma\right)\left(k^{2} d_{3}+\mu+\delta\right)-\delta \gamma\right], \\
p_{1}= & \left(k^{2} d_{2}+\mu+\gamma\right)\left(k^{2} d_{3}+\mu+\delta\right)-\delta \gamma \\
& +\left(k^{2} d_{1}+\mu+\beta I^{*}\right)\left(k^{2} d_{2}+k^{2} d_{3}+2 \mu+\delta+\gamma\right), \\
p_{2}= & \left(k^{2} d_{1}+\mu+\beta I^{*}\right)+\left(k^{2} d_{2}+\mu+\gamma\right)+\left(k^{2} d_{3}+\mu+\delta\right), \\
q_{0}= & -\beta S^{*} e^{-\mu \tau}\left(k^{2} d_{1}+\mu\right)\left(k^{2} d_{3}+\mu+\delta\right), \\
q_{1}= & -\beta S^{*} e^{-\mu \tau}\left(k^{2} d_{1}+k^{2} d_{3}+2 \mu+\delta\right), \\
q_{2}= & -\beta S^{*} e^{-\mu \tau} .
\end{aligned}
$$

When $\tau=0$, (28) becomes

$$
\lambda^{3}+\left(p_{2}+q_{2}\right) \lambda^{2}+\left(p_{1}+q_{1}\right) \lambda+p_{0}+q_{0}=0 .
$$

It is not difficult to verify that

$$
\begin{aligned}
p_{2}+q_{2}> & \mu+\gamma-\beta S^{*}=\frac{\delta \gamma}{\mu+\delta}>0 \\
p_{0}+q_{0}> & \left(k^{2} d_{1}+\mu\right)\left[(\mu+\gamma)\left(k^{2} d_{3}+\mu+\delta\right)-\delta \gamma\right] \\
& -\beta S^{*}\left(k^{2} d_{1}+\mu\right)\left(k^{2} d_{3}+\mu+\delta\right), \\
> & (\mu+\delta)\left(k^{2} d_{1}+\mu\right) \frac{\delta \gamma}{\mu+\delta}-\delta \gamma\left(k^{2} d_{1}+\mu\right)=0, \\
\left(p_{1}+q_{1}\right) & \left(p_{2}+q_{2}\right)-\left(p_{0}+q_{0}\right) \\
> & {\left[\left(k^{2} d_{2}+\mu+\gamma\right)\left(k^{2} d_{3}+\mu+\delta\right)-\delta \gamma\right.} \\
& +\left(k^{2} d_{1}+\mu+\beta I^{*}\right)\left(k^{2} d_{2}+k^{2} d_{3}+2 \mu+\delta+\gamma\right) \\
& \left.-\beta S^{*}\left(k^{2} d_{1}+k^{2} d_{3}+2 \mu+\delta\right)\right] \\
\times & \left(k^{2} d_{2}+k^{2} d_{3}+2 \mu+\delta+\gamma-\beta S^{*}\right)>0,
\end{aligned}
$$

which indicates that all roots $\lambda_{i, 1}, \lambda_{i, 2}$, and $\lambda_{i, 3}$ of (30) have negative real parts.

Next we can conclude that there exists a positive constant $\xi$ such that

$$
\operatorname{Re}\left\{\lambda_{i, 1}\right\}, \operatorname{Re}\left\{\lambda_{i, 2}\right\}, \operatorname{Re}\left\{\lambda_{i, 3}\right\} \leqslant-\xi, \quad i \geqslant 1
$$

In fact, let $\lambda=\mu_{i} v$, then

$$
\begin{aligned}
\lambda^{3}+ & \left(p_{2}+q_{2}\right) \lambda^{2}+\left(p_{1}+q_{1}\right) \lambda+p_{0}+q_{0} \\
& =\mu_{i}^{3} v^{3}+\left(p_{2}+q_{2}\right) \mu_{i}^{2} v^{2}+\left(p_{1}+q_{1}\right) \mu_{i} v+p_{0}+q_{0} \\
& \triangleq h_{i}(v) .
\end{aligned}
$$

Since $\mu_{i} \rightarrow \infty$ as $i \rightarrow \infty$, it yields that

$$
\begin{aligned}
\lim _{i \rightarrow \infty} \frac{h_{i}(v)}{\mu_{i}^{3}}= & v^{3}+\left(d_{1}+d_{2}+d_{3}\right) v^{2} \\
& +\left(d_{1} d_{2}+d_{1} d_{3}+d_{2} d_{3}\right) v+d_{1} d_{2} d_{3} \\
\triangleq & h(v) .
\end{aligned}
$$

It is easy to see that these three roots $v_{1}, v_{2}$, and $v_{3}$ of $h(v)$ have negative real parts from the Routh-Hurwitz criterion again, and thus, there exists a positive constant $\bar{\xi}$ such that

$$
\operatorname{Re}\left\{v_{1}\right\}, \operatorname{Re}\left\{v_{2}\right\}, \operatorname{Re}\left\{v_{3}\right\} \leqslant-\bar{\xi}
$$

By continuity, we see that there exists $i_{0}$ such that the three roots $v_{i, 1}, v_{i, 2}$, and $v_{i, 3}$ of $h_{i}(v)$ satisfy

$$
\operatorname{Re}\left\{v_{i, 1}\right\}, \operatorname{Re}\left\{v_{i, 2}\right\}, \operatorname{Re}\left\{v_{i, 3}\right\} \leqslant-\frac{\bar{\xi}}{2}, \quad i \geqslant i_{0},
$$


and thus,

$$
\operatorname{Re}\left\{\lambda_{i, 1}\right\}, \operatorname{Re}\left\{\lambda_{i, 2}\right\}, \operatorname{Re}\left\{\lambda_{i, 3}\right\} \leqslant-\frac{\mu_{i} \bar{\xi}}{2} \leqslant-\frac{\mu_{i_{0}} \bar{\xi}}{2}, \quad i \geqslant i_{0} .
$$

Let

$$
-\widetilde{\xi}=\max _{1 \leqslant i \leqslant i_{0}}\left\{\operatorname{Re}\left\{\lambda_{i, 1}\right\}, \operatorname{Re}\left\{\lambda_{i, 2}\right\}, \operatorname{Re}\left\{\lambda_{i, 3}\right\}\right\}
$$

then $\widetilde{\xi}$, and (32) holds for $\xi \in\left(\widetilde{\xi}, \mu_{i_{0}} \bar{\xi} / 2\right)$.

Consequently, the spectrum of $\mathscr{L}$, consisting only of eigenvalues, lies in $\operatorname{Re} \lambda \leqslant-\xi$. Hence, the infected steady state is locally asymptotically stable when $\tau=0$.

For $\tau>0$, if $i \omega(\omega>0)$ is a solution of (28), separating the real and imaginary parts, we derive that

$$
\begin{aligned}
& \omega^{3}-p_{1} \omega=q_{1} \omega \cos \omega \tau+\left(q_{2} \omega^{2}-q_{0}\right) \sin \omega \tau \\
& p_{2} \omega^{2}-p_{0}=q_{1} \omega \sin \omega \tau-\left(q_{2} \omega^{2}-q_{0}\right) \cos \omega \tau .
\end{aligned}
$$

Squaring and adding the two equations of (39), it follows that

$$
\begin{aligned}
& \omega^{6}+\left(p_{2}^{2}-2 p_{1}-q_{2}^{2}\right) \omega^{4}+\left(p_{1}^{2}-2 p_{0} p_{2}-q_{1}^{2}+2 q_{0} q_{2}\right) \omega^{2} \\
& +p_{0}^{2}-q_{0}^{2}=0 .
\end{aligned}
$$

By calculation, it is not difficult to verify that, for all $i \geqslant 1$,

$$
\begin{aligned}
p_{2}^{2}-2 & p_{1}-q_{2}^{2} \\
= & \left(k^{2} d_{1}+\mu+\beta I^{*}\right)^{2}+\left(k^{2} d_{2}+\mu+\gamma\right)^{2} \\
& +\left(k^{2} d_{3}+\mu+\delta\right)^{2}+2 \delta \gamma-\left(\beta S^{*} e^{-\mu \tau}\right)^{2} \\
> & (\mu+\gamma)^{2}-\beta^{2} S^{* 2}>0, \\
p_{1}^{2}-2 & p_{0} p_{2}-q_{1}^{2}+2 q_{0} q_{2} \\
> & {\left[\left(k^{2} d_{2}+\mu+\gamma\right)\left(k^{2} d_{3}+\mu+\delta\right)-\delta \gamma\right]^{2} } \\
& -\left(\beta S^{*} e^{-\mu \tau}\right)^{2}\left(k^{2} d_{3}+\mu+\delta\right)^{2} \\
> & \mu^{2}(\mu+\delta+\gamma)^{2}-\left[\beta S^{*} e^{-\mu \tau}(\mu+\delta)\right]^{2}=0, \\
& p_{0}^{2}-q_{0}^{2}=\left(p_{0}-q_{0}\right)\left(p_{0}+q_{0}\right)>0 .
\end{aligned}
$$

Hence, (40) has no positive roots. Therefore, if $\mathscr{R}_{0}>1$, $E^{*}$ is locally asymptotically stable for all $\tau>0$.

From the discussion above, we can obtain the following results.

Theorem 1. For system (5) with initial conditions (6) and homogeneous Neumann conditions (8), if $\mathscr{R}_{0}<1, E_{0}$ is locally asymptotically stable; if $\mathscr{R}_{0}>1, E_{0}$ becomes unstable, and $E^{*}$ is locally asymptotically stable.

\section{Existence of Travelling Waves}

In this section, we investigate the existence of travelling wave solutions to system (5). The technique of the proofs is to use the Schauder's fixed point theorem, the method of upperlower solutions, and its associated cross iteration scheme.

Let $\widehat{S}=1-S$, then system (5) is transformed into (omitting the hat on $S$ for simplicity)

$$
\begin{aligned}
& \frac{\partial S(x, t)}{\partial t}=d_{1} \frac{\partial^{2} S(x, t)}{\partial x^{2}}-\mu S(x, t)+\beta I(x, t)(1-S(x, t)) \\
& \begin{aligned}
\frac{\partial I(x, t)}{\partial t}= & d_{2} \frac{\partial^{2} I(x, t)}{\partial x^{2}}+\beta e^{-\mu \tau} I(x, t-\tau)(1-S(x, t-\tau)) \\
& +\delta R(x, t)-(\mu+\gamma) I(x, t), \\
\frac{\partial R(x, t)}{\partial t}= & d_{3} \frac{\partial^{2} R(x, t)}{\partial x^{2}}+\gamma I(x, t)-(\mu+\delta) R(x, t) .
\end{aligned}
\end{aligned}
$$

A travelling wave solution of (42) is a solution $(S(x, t)$, $I(x, t), R(x, t))$ of the form $S(x, t)=\phi(x+c t), I(x, t)=\varphi(x+$ $c t), R(x, t)=\psi(x+c t)$ where $\phi, \varphi, \psi \in C^{2}(\mathbb{R}, \mathbb{R})$ and $c>0$ is a constant corresponding to the wave speed. On substituting $S(x, t)=\phi(x+c t), I(x, t)=\varphi(x+c t)$, and $R(x, t)=\psi(x+c t)$ denoting the travelling wave coordinate $x+c t$ still by $t$, we derive from (42) that

$$
\begin{aligned}
& d_{1} \phi^{\prime \prime}(t)-c \phi^{\prime}(t)+f_{c 1}\left(\phi_{t}, \varphi_{t}, \psi_{t}\right)=0, \\
& d_{2} \varphi^{\prime \prime}(t)-c \varphi^{\prime}(t)+f_{c 2}\left(\phi_{t}, \varphi_{t}, \psi_{t}\right)=0, \\
& d_{3} \psi^{\prime \prime}(t)-c \psi^{\prime}(t)+f_{c 3}\left(\phi_{t}, \varphi_{t}, \psi_{t}\right)=0,
\end{aligned}
$$

where

$f_{c 1}\left(\phi_{t}, \varphi_{t}, \psi_{t}\right)=-\mu \phi(t)+\beta \varphi(t)(1-\phi(t))$,

$f_{c 2}\left(\phi_{t}, \varphi_{t}, \psi_{t}\right)$

$=\beta e^{-\mu \tau} \varphi(t-c \tau)(1-\phi(t-c \tau))+\delta \psi(t)-(\mu+\gamma) \varphi(t)$,

$f_{c 3}\left(\phi_{t}, \varphi_{t}, \psi_{t}\right)=\gamma \varphi(t)-(\mu+\delta) \psi(t)$.

Equation (43) will be solved subject to the following conditions:

$$
\begin{gathered}
\operatorname{Lim}_{t \rightarrow-\infty}(\phi(t), \varphi(t), \psi(t))=(0,0,0), \\
\lim _{t \rightarrow+\infty}(\phi(t), \varphi(t), \psi(t))=\left(k_{1}, k_{2}, k_{3}\right) \triangleq\left(1-S^{*}, I^{*}, R^{*}\right) .
\end{gathered}
$$

We define the upper and lower solutions of system (43) as follows.

Definition 2. A pair of continuous functions $\bar{\Phi}=(\bar{\phi}, \bar{\varphi}, \bar{\psi})$ and $\underline{\Phi}=(\phi, \varphi, \psi)$ are called a pair of upper-lower solutions of system $(43)$ if $\bar{\Phi}$ and $\underline{\Phi}$ are twice differential almost 
everywhere in $\mathbb{R}$ and they are essentially bounded on $\mathbb{R}$, and there hold

$$
\begin{aligned}
& d_{1} \bar{\phi}^{\prime \prime}(t)-c \bar{\phi}^{\prime}(t)+f_{c 1}\left(\bar{\phi}_{t}, \bar{\varphi}_{t}, \bar{\psi}_{t}\right) \leqslant 0, \quad \text { a.e. in } \mathbb{R}, \\
& d_{2} \bar{\varphi}^{\prime \prime}(t)-c \bar{\varphi}^{\prime}(t)+f_{c 2}\left(\underline{\phi}_{t}, \bar{\varphi}_{t}, \bar{\psi}_{t}\right) \leqslant 0, \quad \text { a.e. in } \mathbb{R}, \\
& d_{3} \bar{\psi}^{\prime \prime}(t)-c \bar{\psi}^{\prime}(t)+f_{c 3}\left(\bar{\phi}_{t}, \bar{\varphi}_{t}, \bar{\psi}_{t}\right) \leqslant 0, \quad \text { a.e. in } \mathbb{R}, \\
& d_{1} \underline{\phi}^{\prime \prime}(t)-c \underline{\phi}^{\prime}(t)+f_{c 1}\left(\underline{\phi}_{t}, \underline{\varphi}_{t}, \underline{\psi}_{t}\right) \geqslant 0, \quad \text { a.e. in } \mathbb{R}, \\
& d_{2} \underline{\varphi}^{\prime \prime}(t)-c \underline{\varphi}^{\prime}(t)+f_{c 2}\left(\bar{\phi}_{t}, \underline{\varphi}_{t}, \underline{\psi}_{t}\right) \geqslant 0, \\
& d_{3} \underline{\psi}^{\prime \prime}(t)-c \underline{\psi}^{\prime}(t)+f_{c 3}\left(\underline{\phi}_{t}, \underline{\varphi}_{t}, \underline{\psi}_{t}\right) \geqslant 0, \quad \text { a.e. in } \mathbb{R},
\end{aligned}
$$

As to $f_{c 1}, f_{c 2}$, and $f_{c 3}$, we have the following.

Lemma 3. $f_{c 1}, f_{c 2}$, and $f_{c 3}$ satisfy the following partial quasimonotonicity conditions.

(C1) There exist three positive constants $\beta_{1}, \beta_{2}, \beta_{3}$ such that $f_{c 1}\left(\phi_{1}, \varphi_{1}, \psi_{1}\right)-f_{c 1}\left(\phi_{2}, \varphi_{2}, \psi_{2}\right)+\beta_{1}\left[\phi_{1}(0)-\phi_{2}(0)\right] \geqslant 0$, $f_{c 2}\left(\phi_{1}, \varphi_{1}, \psi_{1}\right)-f_{c 2}\left(\phi_{1}, \varphi_{2}, \psi_{2}\right)+\beta_{2}\left[\varphi_{1}(0)-\varphi_{2}(0)\right] \geqslant 0$,

$$
\begin{gathered}
f_{c 2}\left(\phi_{1}, \varphi_{1}, \psi_{1}\right)-f_{c 2}\left(\phi_{2}, \varphi_{1}, \psi_{1}\right) \leqslant 0, \\
f_{c 3}\left(\phi_{1}, \varphi_{1}, \psi_{1}\right)-f_{c 3}\left(\phi_{2}, \varphi_{2}, \psi_{2}\right)+\beta_{3}\left[\psi_{1}(0)-\psi_{2}(0)\right] \geqslant 0,
\end{gathered}
$$

where $\phi_{i}, \varphi_{i}, \psi_{i} \in C([-\tau, 0], R), i=1,2$ with $(0,0,0) \leqslant$ $\left(\phi_{2}, \varphi_{2}, \psi_{2}\right) \leqslant\left(\phi_{1}, \varphi_{1}, \psi_{1}\right) \leqslant\left(M_{1}, M_{2}, M_{3}\right), M_{j}>$ $k_{j}(j=1,2,3)$ are positive constants.

Proof. It is not difficult to verify that

$$
\begin{aligned}
& f_{c 1}\left(\phi_{1}, \varphi_{1}, \psi_{1}\right)-f_{c 1}\left(\phi_{2}, \varphi_{2}, \psi_{2}\right) \\
&= \beta\left(1-\phi_{1}(0)\right) \varphi_{1}(0)-\mu \phi_{1}(0)+\mu \phi_{2}(0) \\
&+\beta\left(1-\phi_{2}(0)\right) \varphi_{2}(0) \\
& \geqslant-\left(\mu+\beta \phi_{2}(0)\right)\left(\phi_{1}(0)-\phi_{2}(0)\right), \\
& f_{c 2}\left(\phi_{1}, \varphi_{1}, \psi_{1}\right)-f_{c 2}\left(\phi_{1}, \varphi_{2}, \psi_{2}\right) \\
&=\beta e^{-\mu \tau}\left(1-\phi_{1}(-\tau)\right)\left(\varphi_{1}(-\tau)-\varphi_{2}(-\tau)\right) \\
& \quad+\delta\left(\psi_{1}(0)-\psi_{2}(0)\right) \\
& \quad-(\mu+\gamma)\left(\varphi_{1}(0)-\varphi_{2}(0)\right) \\
& \geqslant-(\mu+\gamma)\left(\varphi_{1}(0)-\varphi_{2}(0)\right), \\
& f_{c 2}\left(\phi_{1},\right.\left.\varphi_{1}, \psi_{1}\right)-f_{c 2}\left(\phi_{2}, \varphi_{1}, \psi_{1}\right) \\
&= \beta e^{-\mu \tau} \varphi_{1}(-\tau)\left(\phi_{2}(-\tau)-\phi_{1}(-\tau)\right) \leqslant 0, \\
& f_{c 3}\left(\phi_{1},\right.\left.\varphi_{1}, \psi_{1}\right)-f_{c 3}\left(\phi_{2}, \varphi_{2}, \psi_{2}\right) \\
& \geqslant-(\mu+\delta)\left[\psi_{1}(0)-\psi_{2}(0)\right] \geqslant 0 .
\end{aligned}
$$

Let $\beta_{1}=\mu+\beta \phi_{2}(0), \beta_{2}=\mu+\gamma$, and $\beta_{3}=\mu+\delta$, then the proof is completed.

For the constants $\beta_{1}, \beta_{2}, \beta_{3}$ above, define

$$
\begin{gathered}
H_{1}(\phi, \varphi, \psi)(t)=f_{c 1}\left(\phi_{t}, \varphi_{t}, \psi_{t}\right)+\beta_{1} \phi(t), \\
H_{2}(\phi, \varphi, \psi)(t)=f_{c 2}\left(\phi_{t}, \varphi_{t}, \psi_{t}\right)+\beta_{2} \varphi(t), \\
H_{3}(\phi, \varphi, \psi)(t)=f_{c 3}\left(\phi_{t}, \varphi_{t}, \psi_{t}\right)+\beta_{3} \psi(t), \\
F_{1}(\phi, \varphi, \psi)(t) \\
=\frac{1}{d_{1}\left(\lambda_{2}-\lambda_{1}\right)} \\
\quad \times\left[\int_{-\infty}^{t} e^{\lambda_{1}(t-s)} H_{1}(\phi, \varphi, \psi)(s) \mathrm{d} s\right. \\
\left.\quad+\int_{t}^{\infty} e^{\lambda_{2}(t-s)} H_{1}(\phi, \varphi, \psi)(s) \mathrm{d} s\right],
\end{gathered}
$$

$F_{2}(\phi, \varphi, \psi)(t)$

$$
\begin{aligned}
& =\frac{1}{d_{2}\left(\lambda_{4}-\lambda_{3}\right)} \\
& \quad \times\left[\int_{-\infty}^{t} e^{\lambda_{3}(t-s)} H_{2}(\phi, \varphi, \psi)(s) \mathrm{d} s\right. \\
& \left.\quad+\int_{t}^{\infty} e^{\lambda_{4}(t-s)} H_{2}(\phi, \varphi, \psi)(s) \mathrm{d} s\right],
\end{aligned}
$$

$F_{3}(\phi, \varphi, \psi)(t)$

$$
\begin{aligned}
& =\frac{1}{d_{3}\left(\lambda_{6}-\lambda_{5}\right)} \\
& \quad \times\left[\int_{-\infty}^{t} e^{\lambda_{5}(t-s)} H_{3}(\phi, \varphi, \psi)(s) \mathrm{d} s\right. \\
& \left.\quad+\int_{t}^{\infty} e^{\lambda_{6}(t-s)} H_{3}(\phi, \varphi, \psi)(s) \mathrm{d} s\right],
\end{aligned}
$$

where $(0,0,0) \leqslant(\phi, \varphi, \psi) \leqslant\left(k_{1}, k_{2}, k_{3}\right)$ and

$$
\begin{array}{ll}
\lambda_{1}=\frac{c-\sqrt{c^{2}+4 \beta_{1} d_{1}}}{2 d_{1}}, & \lambda_{2}=\frac{c+\sqrt{c^{2}+4 \beta_{1} d_{1}}}{2 d_{1}}, \\
\lambda_{3}=\frac{c-\sqrt{c^{2}+4 \beta_{2} d_{2}}}{2 d_{2}}, & \lambda_{4}=\frac{c+\sqrt{c^{2}+4 \beta_{2} d_{2}}}{2 d_{2}}, \\
\lambda_{5}=\frac{c-\sqrt{c^{2}+4 \beta_{3} d_{3}}}{2 d_{3}}, & \lambda_{6}=\frac{c+\sqrt{c^{2}+4 \beta_{3} d_{3}}}{2 d_{3}} .
\end{array}
$$

Define the following profile set:

$$
\begin{gathered}
\Gamma((\underline{\phi}, \underline{\varphi}, \underline{\psi}),(\bar{\phi}, \bar{\varphi}, \bar{\psi})) \\
=\left\{(\phi, \varphi, \psi) \in C\left(\mathbb{R}, \mathbb{R}^{3}\right) \mid(\underline{\phi}, \underline{\varphi}, \underline{\psi})\right. \\
\leqslant(\phi, \varphi, \psi) \leqslant(\bar{\phi}, \bar{\varphi}, \bar{\psi})\} .
\end{gathered}
$$

From Lemmas 3.1-3.6 of [11], we have the following results. 
Lemma 4. $F(\Gamma((\underline{\phi}, \underline{\varphi}, \underline{\psi}),(\bar{\phi}, \bar{\varphi}, \bar{\psi}))) \subset \Gamma((\underline{\phi}, \underline{\varphi}, \underline{\psi}),(\bar{\phi}, \bar{\varphi}, \bar{\psi}))$, where $F=\left(F_{1}, F_{2}\right)$.

Lemma 5. $F: \Gamma((\phi, \varphi, \psi),(\bar{\phi}, \bar{\varphi}, \bar{\psi})) \rightarrow \Gamma((\phi, \varphi, \psi),(\bar{\phi}, \bar{\varphi}, \bar{\psi}))$ is compact.

From Lemmas 4 and 5, we see that the existence of travelling wave solutions for system (5) follows from the existence of a pair of upper and lower solutions $(\bar{\phi}, \bar{\varphi}, \bar{\psi})$ and $(\phi, \varphi, \psi)$ of (43) satisfying the following conditions.

(C2) $(0,0,0) \leqslant(\phi(t), \varphi(t), \psi(t)) \leqslant(\bar{\phi}(t), \bar{\varphi}(t), \bar{\psi}(t)) \leqslant\left(M_{1}\right.$, $\left.M_{2}, M_{3}\right), t \in \mathbb{R}$.

(C3) $\lim _{t \rightarrow-\infty}(\phi(t), \varphi(t), \psi(t))=(0,0,0), \lim _{t \rightarrow+\infty}(\bar{\phi}(t)$, $\bar{\varphi}(t), \bar{\psi}(t))=\left(k_{1}, k_{2}, \bar{k}_{3}\right)$.

Let $d_{1}=d_{2}=d_{3}$ and $c>c^{*} \triangleq \max \left\{2 \sqrt{d_{1} \beta k_{2}}\right.$, $\left.2 \sqrt{d_{2} \beta k_{1} k_{2} e^{-\mu \tau}}\right\}$, then there exists $\lambda_{0}$ such that

$$
d_{1} \lambda_{0}^{2}-c \lambda_{0}+\beta k_{2}<0, \quad d_{2} \lambda_{0}^{2}-c \lambda_{0}+\beta k_{1} k_{2} e^{-\mu \tau}<0 .
$$

We can choose $\varepsilon_{i}>0 \quad(i=1,2, \ldots, 6)$ such that

$$
\begin{gathered}
\beta \varepsilon_{2}\left(1-k_{1}\right)-\beta \varepsilon_{1} k_{2}-\mu \varepsilon_{1}<0, \\
\beta e^{-\mu \tau}\left[\varepsilon_{2}\left(1-k_{1}+\varepsilon_{4}\right)+k_{2} \varepsilon_{4}\right]+\delta \varepsilon_{3}-(\mu+\gamma) \varepsilon_{2}<0, \\
\gamma \varepsilon_{2}-(\mu+\delta) \varepsilon_{3}<0, \\
\beta \varepsilon_{5}\left(1-k_{1}+\varepsilon_{4}\right)-\left(\beta k_{2}+\mu\right) \varepsilon_{4}<0, \\
\delta \varepsilon_{6}-(\mu+\gamma) \varepsilon_{5}<0, \\
\gamma \varepsilon_{5}-(\mu+\delta) \varepsilon_{6}<0 .
\end{gathered}
$$

For the above constants and suitable $t_{i} \quad(i=1,2, \ldots, 6)$ satisfying $t_{4}>0$ and $t_{5}<t_{6}$, we define the continuous functions $\Phi(t)=\left(\phi_{1}(t), \varphi_{1}(t), \psi_{1}(t)\right)$ and $\Psi(t)=\left(\phi_{2}(t), \varphi_{2}(t)\right.$, $\left.\psi_{2}(t)\right)$ as follows:

$$
\begin{aligned}
& \phi_{1}(t)= \begin{cases}k_{1} e^{\lambda_{0} t}, & t \leqslant t_{1}, \\
k_{1}+\varepsilon_{1} e^{-\lambda t}, & t>t_{1},\end{cases} \\
& \varphi_{1}(t)= \begin{cases}k_{2} e^{\lambda_{0} t}, & t \leqslant t_{2}, \\
k_{2}+\varepsilon_{2} e^{-\lambda t}, & t>t_{2},\end{cases} \\
& \psi_{1}(t)= \begin{cases}k_{3} e^{\lambda_{0} t}, & t \leqslant t_{3}, \\
k_{3}+\varepsilon_{3} e^{-\lambda t}, & t>t_{3},\end{cases} \\
& \phi_{2}(t)= \begin{cases}0, & t \leqslant t_{4}, \\
k_{1}-\varepsilon_{4} e^{-\lambda t}, & t>t_{4},\end{cases} \\
& \varphi_{2}(t)= \begin{cases}0, & t \leqslant t_{5}, \\
k_{2}-\varepsilon_{5} e^{-\lambda t}, & t>t_{5},\end{cases} \\
& \psi_{2}(t)= \begin{cases}0, & t \leqslant t_{6}, \\
k_{3}-\varepsilon_{6} e^{-\lambda t}, & t>t_{6} .\end{cases}
\end{aligned}
$$

Lemma 6. $\Phi(t)=\left(\phi_{1}(t), \varphi_{1}(t), \psi_{1}(t)\right)$ is an upper solution of system (43).

Proof. Denote

$$
\begin{aligned}
p_{1}(t)= & d_{1} \phi_{1}^{\prime \prime}(t)-c \phi_{1}^{\prime}(t)+\beta \varphi_{1}(t)\left(1-\phi_{1}(t)\right)-\mu \phi_{1}(t), \\
p_{2}(t)= & d_{2} \varphi_{1}^{\prime \prime}(t)-c \varphi_{1}^{\prime}(t)+\beta e^{-\mu \tau} \varphi_{1}(t-c \tau)\left(1-\phi_{2}(t-c \tau)\right) \\
& +\delta \psi_{1}(t)-(\mu+\gamma) \varphi_{1}(t), \\
p_{3}(t)= & d_{3} \psi_{1}^{\prime \prime}(t)-c \psi_{1}^{\prime}(t)+\gamma \varphi_{1}(t)-(\mu+\delta) \psi_{1}(t) .
\end{aligned}
$$

$$
\begin{aligned}
& \text { If } t \leqslant t_{1}, \phi_{1}(t)=k_{1} e^{\lambda_{0} t}, \varphi_{1}(t) \leqslant k_{2} e^{\lambda_{0} t}, \text { then } \\
& \begin{aligned}
p_{1}(t) \leqslant & d_{1} k_{1} \lambda_{0}^{2} e^{\lambda_{0} t}-c k_{1} \lambda_{0} e^{\lambda_{0} t}+\beta\left(1-\phi_{1}(t)\right) k_{2} e^{\lambda_{0} t} \\
& -\mu k_{1} e^{\lambda_{0} t} \\
\leqslant & k_{1} e^{\lambda_{0} t}\left(d_{1} \lambda_{0}^{2}-c \lambda_{0}+\beta k_{2}\right)<0 .
\end{aligned}
\end{aligned}
$$

If $t>t_{1}, \phi_{1}(t)=k_{1}+\varepsilon_{1} e^{-\lambda t}, \varphi_{1}(t) \leqslant k_{2}+\varepsilon_{2} e^{-\lambda t}$. It follows that

$$
p_{1}(t) \leqslant e^{-\lambda t}\left[d_{1} \varepsilon_{1} \lambda^{2}+c \varepsilon_{1} \lambda+\beta \varepsilon_{2}\left(1-k_{1}\right)-\beta \varepsilon_{1} k_{2}-\mu \varepsilon_{1}\right] .
$$

Since $\beta\left(1-k_{1}\right) \varepsilon_{2}-\beta \varepsilon_{1} k_{2}-\mu \varepsilon_{1}<0$, then there exists a $\lambda_{1}^{*}>0$ such that $p_{1}(t)<0$ for all $\lambda \in\left(0, \lambda_{1}^{*}\right)$.

If $t \leqslant t_{2}, \varphi_{1}(t)=k_{2} e^{\lambda_{0} t}, \varphi_{1}(t-c \tau)=k_{2} e^{\lambda_{0}(t-c \tau)}, \psi_{1}(t) \leqslant$ $k_{3} e^{\lambda_{0} t}$, then

$$
\begin{aligned}
p_{2}(t) & \leqslant k_{2} e^{\lambda_{0} t}\left[d_{2} \lambda_{0}^{2}-c \lambda_{0}+\beta e^{-\left(\mu+c \lambda_{0}\right) \tau}+\frac{\delta k_{3}}{k_{2}}-(\mu+\gamma)\right] \\
& \leqslant k_{2} e^{\lambda_{0} t}\left(d_{2} \lambda_{0}^{2}-c \lambda_{0}+\beta k_{1} k_{2} e^{-\mu \tau}\right)<0 .
\end{aligned}
$$

If $t>t_{2}$, we obtain that

$$
\begin{aligned}
p_{2}(t) \leqslant e^{-\lambda t}\left\{d_{2} \varepsilon_{2} \lambda^{2}+c \varepsilon_{2} \lambda\right. \\
+\beta e^{-(\mu-c \lambda) \tau} \\
\times\left[\varepsilon_{2}\left(1-k_{1}+\varepsilon_{4} e^{-\lambda(t-c \tau)}\right)+k_{2} \varepsilon_{4}\right] \\
\left.+\delta \varepsilon_{3}-(\mu+\gamma) \varepsilon_{2}\right\} \\
\leqslant e^{-\lambda t}\left[d_{2} \varepsilon_{2} \lambda^{2}+c \varepsilon_{2} \lambda+I_{2}(\lambda)\right],
\end{aligned}
$$

where $I_{1}(\lambda)=\beta e^{-(\mu-c \lambda) \tau}\left[\varepsilon_{2}\left(1-k_{1}+\varepsilon_{4}\right)+k_{2} \varepsilon_{4}\right]+\delta \varepsilon_{3}-(\mu+\gamma) \varepsilon_{2}$, then $I_{1}(0)<0$ indicates that there exists a $\lambda_{2}^{*}>0$ such that $p_{2}(t)<0$ for all $\lambda \in\left(0, \lambda_{2}^{*}\right)$.

If $t \leqslant t_{3}, \varphi_{1}(t) \leqslant k_{2} e^{\lambda_{0} t}, \psi_{1}(t)=k_{3} e^{\lambda_{0} t}$, then

$$
\begin{aligned}
p_{3}(t) & \leqslant e^{\lambda_{0} t}\left[k_{3} d_{3} \lambda_{0}^{2}-c k_{3} \lambda_{0}+\gamma k_{2}-(\mu+\delta) k_{3}\right] \\
& =k_{3} \lambda_{0} e^{\lambda_{0} t}\left(d_{3} \lambda_{0}-c\right)<0 .
\end{aligned}
$$


If $t>t_{3}, \varphi_{1}(t) \leqslant k_{2}+\varepsilon_{2} e^{-\lambda t}, \psi_{1}(t)=k_{3}+\varepsilon_{3} e^{-\lambda t}$, then it follows

$$
p_{3}(t) \leqslant e^{-\lambda t}\left[d_{3} \varepsilon_{3} \lambda^{2}+c \varepsilon_{3} \lambda+\gamma \varepsilon_{2}-(\mu+\delta) \varepsilon_{3}\right] .
$$

Note that $\gamma \varepsilon_{2}-(\mu+\delta) \varepsilon_{3}<0$, then there exists a $\lambda_{3}^{*}>0$ such that $p_{3}(t)<0$ for all $\lambda \in\left(0, \lambda_{3}^{*}\right)$.

Clearly, for all $\lambda \in\left(0, \min \left\{\lambda_{i}^{*}, i=1,2,3\right\}\right), p_{i}(t)<0(i=$ $1,2,3)$. This completes the proof.

Lemma 7. $\Psi(t)=\left(\phi_{2}(t), \varphi_{2}(t), \psi_{2}(t)\right)$ is a lower solution of system (43).

Proof. Denote

$$
\begin{aligned}
q_{1}(t)= & d_{1} \phi_{2}^{\prime \prime}(t)-c \phi_{2}^{\prime}(t)+\beta \varphi_{2}(t)\left(1-\phi_{2}(t)\right)-\mu \phi_{2}(t), \\
q_{2}(t)= & d_{2} \varphi_{2}^{\prime \prime}(t)-c \varphi_{2}^{\prime}(t)+\beta e^{-\mu \tau} \varphi_{2}(t-c \tau)\left(1-\phi_{1}(t-c \tau)\right) \\
& +\delta \psi_{2}(t)-(\mu+\gamma) \varphi_{2}(t), \\
q_{3}(t)= & d_{3} \psi_{2}^{\prime \prime}(t)-c \psi_{2}^{\prime}(t)+\gamma \varphi_{2}(t)-(\mu+\delta) \psi_{2}(t) .
\end{aligned}
$$

$$
\begin{aligned}
& \text { If } t \leqslant t_{4}, \phi_{2}(t)=0, q_{1}(t)=\beta \varphi_{2}(t) \geqslant 0 . \\
& \quad \text { If } t>t_{4}, \phi_{2}(t)=k_{1}-\varepsilon_{4} e^{-\lambda t}, \varphi_{2}(t) \geqslant k_{2}-\varepsilon_{5} e^{-\lambda t}, \text { then } \\
& q_{1}(t) \geqslant-\varepsilon_{4} e^{-\lambda t}\left[d_{1} \lambda^{2}+c \lambda+\frac{\beta \varepsilon_{5}\left(1-k_{1}+\varepsilon_{4}\right)}{\varepsilon_{4}}-\beta k_{2}-\mu\right] .
\end{aligned}
$$

Note that $\beta \varepsilon_{5}\left(1-k_{1}+\varepsilon_{4}\right) / \varepsilon_{4}-\beta k_{2}-\mu<0$, then there exists a $\lambda_{4}^{*}>0$ such that $q_{1}(t)>0$ for all $\lambda \in\left(0, \lambda_{4}^{*}\right)$.

If $t \leqslant t_{5}, q_{2}(t)=0$. If $t>t_{5}, \varphi_{2}(t)=k_{2}-\varepsilon_{5} e^{-\lambda t}, \varphi_{2}(t-c \tau) \geqslant$ $k_{2}-\varepsilon_{5} e^{-\lambda(t-c \tau)}, \phi_{1}(t-c \tau) \leqslant k_{1}+\varepsilon_{1} e^{-\lambda(t-c \tau)}, \psi_{2}(t) \geqslant k_{3}-\varepsilon_{6} e^{-\lambda t}$. It follows that

$$
q_{2}(t) \geqslant-e^{-\lambda t}\left\{d_{2} \varepsilon_{5} \lambda^{2}+c \varepsilon_{5} \lambda+\delta \varepsilon_{6}-(\mu+\gamma) \varepsilon_{5}\right\}
$$

Since $\delta \varepsilon_{6}-(\mu+\gamma) \varepsilon_{5}<0$, then there exists a $\lambda_{5}^{*}>0$ such that $q_{2}(t)>0$ for all $\lambda \in\left(0, \lambda_{5}^{*}\right)$.

If $t \leqslant t_{6}, \psi_{2}(t)=0, q_{3}(t)=\gamma \varphi_{2}(t) \geqslant 0$. If $t>t_{6}, \varphi_{2}(t)=$ $k_{2}-\varepsilon_{5} e^{-\lambda t}, \psi_{2}(t)=k_{3}-\varepsilon_{6} e^{-\lambda t}$, then

$$
q_{3}(t) \geqslant-\varepsilon_{6} e^{-\lambda t}\left[d_{3} \lambda^{2}+c \lambda+\frac{\gamma \varepsilon_{5}}{\varepsilon_{6}}-(\mu+\delta)\right] .
$$

Note that $\gamma \varepsilon_{5} / \varepsilon_{6}-(\mu+\delta)<0$, then there exists a $\lambda_{6}^{*}>0$ such that $q_{3}(t)>0$ for all $\lambda \in\left(0, \lambda_{6}^{*}\right)$.

Clearly, for all $\lambda \in\left(0, \min \left\{\lambda_{i}^{*}, i=4,5,6\right\}\right), q_{i}(t)>0(i=$ $1,2,3)$. This completes the proof.
Combining Lemmas 3-7 with the Schauder's fixed point theorem, we know that there exists a fixed point $\left(\phi^{*}(t)\right.$, $\left.\varphi^{*}(t), \psi^{*}(t)\right)$ of $F$ in $\Gamma((\phi, \varphi, \psi),(\bar{\phi}, \bar{\varphi}))$, which gives a solution of (43). Furthermore, from (C3), we can obtain that

$$
\begin{array}{r}
\operatorname{Lim}_{t \rightarrow-\infty}\left(\phi^{*}(t), \varphi^{*}(t), \psi^{*}(t)\right)=(0,0,0), \\
\lim _{t \rightarrow+\infty}\left(\phi^{*}(t), \varphi^{*}(t), \psi^{*}(t)\right)=\left(k_{1}, k_{2}, k_{3}\right),
\end{array}
$$

which indicates that the fixed point satisfies the asymptotic boundary conditions (45). Therefore, there exists a travelling wave solution for system (42) connecting the steady state $(0,0,0)$ and $\left(k_{1}, k_{2}, k_{3}\right)$, and accordingly, we have the following conclusion.

Theorem 8. Suppose $\mathscr{R}_{0}>1$ and $d_{1}=d_{2}=d_{3}$. For every $c>c^{*}$, system (5) always has a travelling wave solution with speed $c$ connecting the disease-free steady state $E_{0}(1,0,0)$ and the endemic steady state $E^{*}\left(S^{*}, I^{*}, R^{*}\right)$.

\section{Numerical Simulations}

In this section, focusing on the travelling wave solutions of system (5), we perform some numerical simulations.

Firstly, we illustrate the results stated in Theorems 1 and 8. As in [6], we take parameter values motivated by bovine tuberculosis in a cattle herd, with time unit of one year. It is assumed that the exposed period is 9 months (i.e., $\tau=0.75$ ). Taking $\mu=0.1, \delta=\gamma=0.5$ (equal periods of infection and recovery before relapse), $\beta=0.6$, and $d_{1}=d_{2}=d_{3}=0.01$, system (5) with above coefficients has a unique endemic equilibrium $E^{*}(0.3294,0.3394,0.2828)$. For convenience, we truncate the spatial domain $\mathbb{R}$ by $[-10,10]$. It is easy to show that $\mathscr{R}_{0}=3.0363>1$. If we use the following trivial functions as initial conditions:

$$
\begin{gathered}
(S(x, t), I(x, t), R(x, t))=(0.9,0.1,0), \\
-10 \leqslant x \leqslant 10, \quad-\tau \leqslant t \leqslant 0,
\end{gathered}
$$

then the numerical simulations shown in Figures 1(a)-1(c) indicate that the endemic steady state $E^{*}$ is stable. If we change the initial conditions, $E^{*}$ keeps stable (see Figure $1(\mathrm{~d})$; for simplicity, we show only the solutions of $I(x, t))$, which indicates that $E^{*}$ is in fact globally asymptotically stable, although we fail to give a proof theoretically at present.

Now, to illustrate the existence of travelling wave solutions, we use the following piecewise functions as initial conditions:

$$
\begin{aligned}
& (S(x, t), I(x, t), R(x, t)) \\
& \quad= \begin{cases}(1,0,0), & -10 \leqslant x<0,-\tau \leqslant t \leqslant 0, \\
E^{*}, & 0 \leqslant x \leqslant 10,-\tau \leqslant t \leqslant 0 .\end{cases}
\end{aligned}
$$




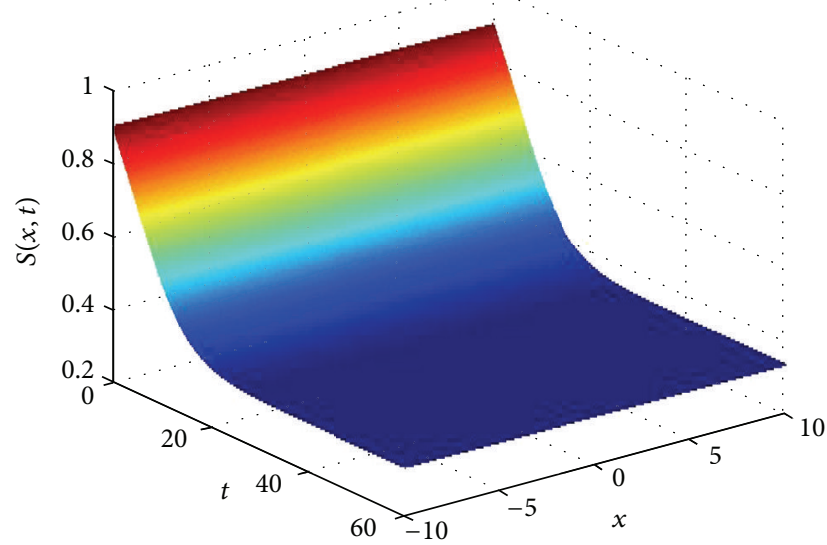

(a)

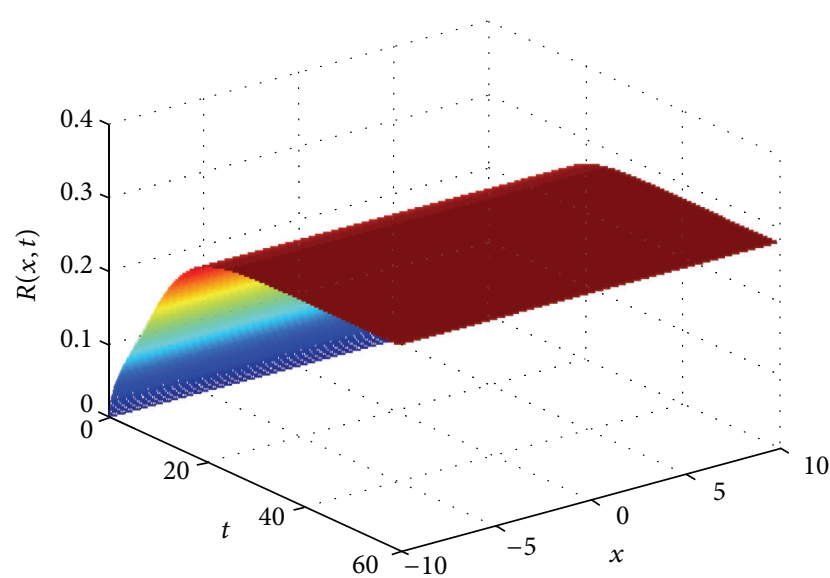

(c)

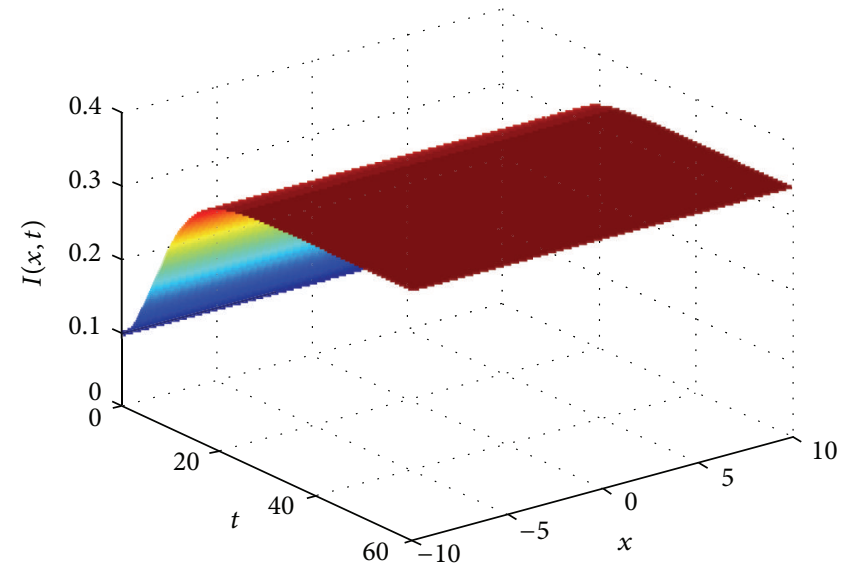

(b)

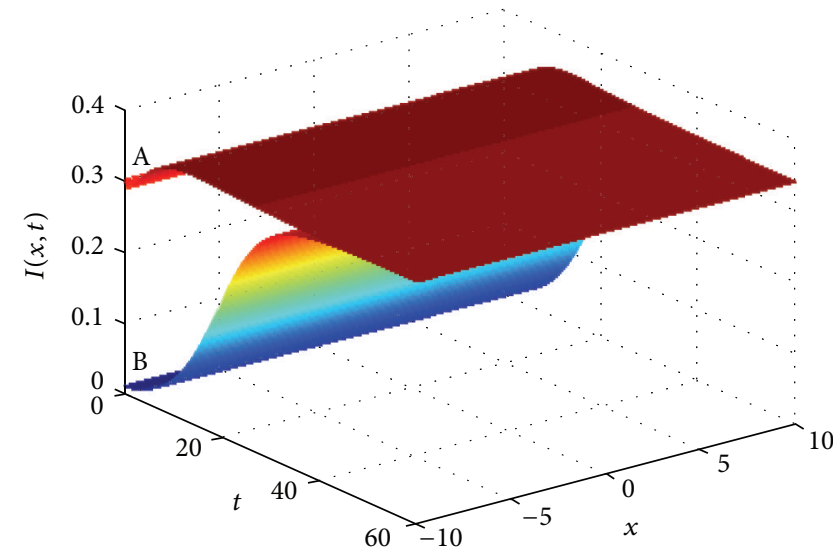

(d)

FIGURE 1: The temporal solution found by numerical integration of system (5) with parameters $\mu=0.1, \delta=\gamma=0.5, \beta=0.6, \tau=0.75, d_{1}=$ $d_{2}=d_{3}=0.01$, and the initial conditions are $(0.9,0.1,0)$ in $(\mathrm{a})-(\mathrm{c}),(0.6,0.3,0.1)$ in $(\mathrm{A})$ of $(\mathrm{d})$, and $(0.99,0.01,0)$ in $(\mathrm{B})$ of $(\mathrm{d})$.

The numerical simulations shown in Figure 2 indicate that system (5) has a travelling wave solution with speed $c \approx 0.07$ connecting $E_{0}$ and $E^{*}$.

Secondly, we address the effects of parameters on the dynamics of system (5). For this part, the following parameter values will be fixed:

$$
\mu=0.1, \quad \gamma=0.5
$$

and the initial conditions are the same as in (68).

We first investigate the impact of the diffusion rates. To this end, we fix $\delta=0.5, \beta=0.6, \tau=0.75$, and let the diffusivity $d_{1}, d_{2}$, and $d_{3}$ vary. In this case, the values of $\mathscr{R}_{0}$ and $E^{*}$ are the same as those in Figure 2 (Note that $\mathscr{R}_{0}$ and $E^{*}$ are independent of the diffusion rates). Figure 3 is the simulation results of (5). In Figure 3(a), $d_{1}=d_{2}=d_{3}=0.01$, but in Figures 3(b)-3(d), we increase the values of $d_{1}, d_{2}$, and $d_{3}$ to 0.04 , respectively. By contrasting these figures, we can see that the travelling waves in Figures 3(c) and 3(d) are much fast than those in Figure 3(a), but the wave speed in
Figure 3(b) is just the same as that in Figure 3(a), which indicates that both the diffusion rates of infective and recovered individuals can advance the time to arrive at the infection steady state, but the diffusion rate of susceptible individuals cannot. Even so, there is an obvious difference between the wave profiles in Figure 3(b) and the other three ones: the former has a hump in wave profile for $I$, but the latter does not seem to show that any more; that is, the diffusion rate of susceptible individuals could lead to nonmonotone travelling waves. In biological meaning, large diffusion of susceptible individuals may enhance the accumulative effect of infection.

Next, we study the impact of $\tau$, that is, the average latent period of the disease. To this end, we fix $\delta=0.5, \beta=0.6$, $d_{1}=0.02$, and $d_{2}=d_{3}=0.01$, and let $\tau$ vary. In this case, the values of $\mathscr{R}_{0}$ and $E^{*}$ are different. (note that both $\mathscr{R}_{0}$ and $E^{*}$ are decreasing in $\tau$ ). Figure 4 is the simulation results of (5). In Figure 4(a), $\tau=0.75$, but in Figure 4(b), we increase $\tau$ to 7.5. By contrasting the two figures, we can see that the travelling wave speed in Figure 4(b) is less than that in Figure 4(a), 


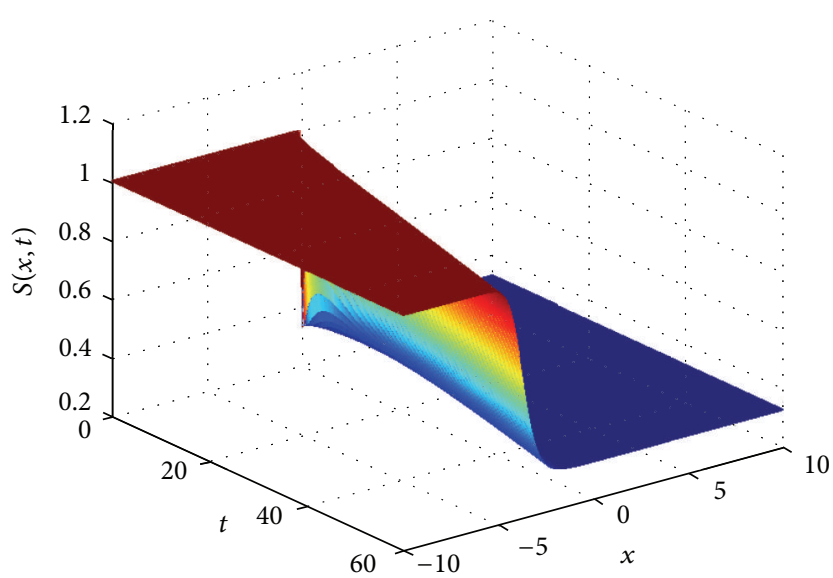

(a)

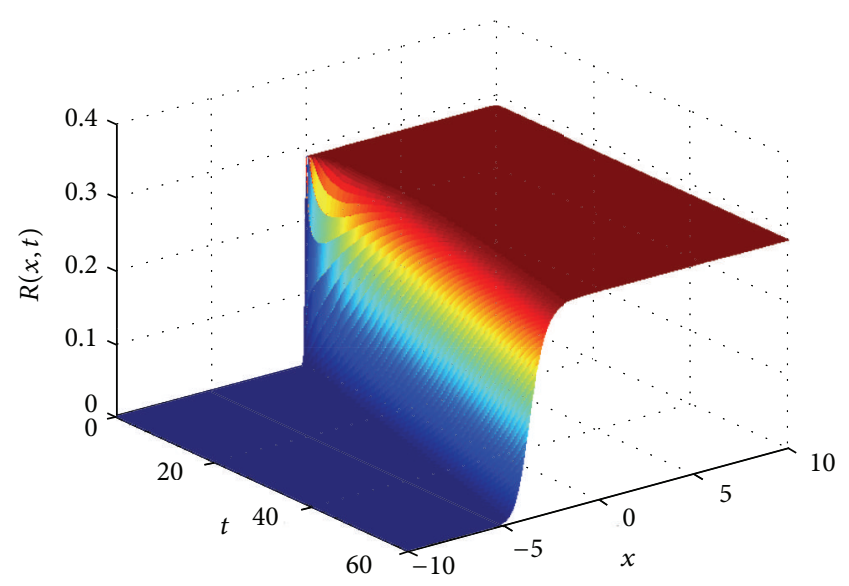

(c)

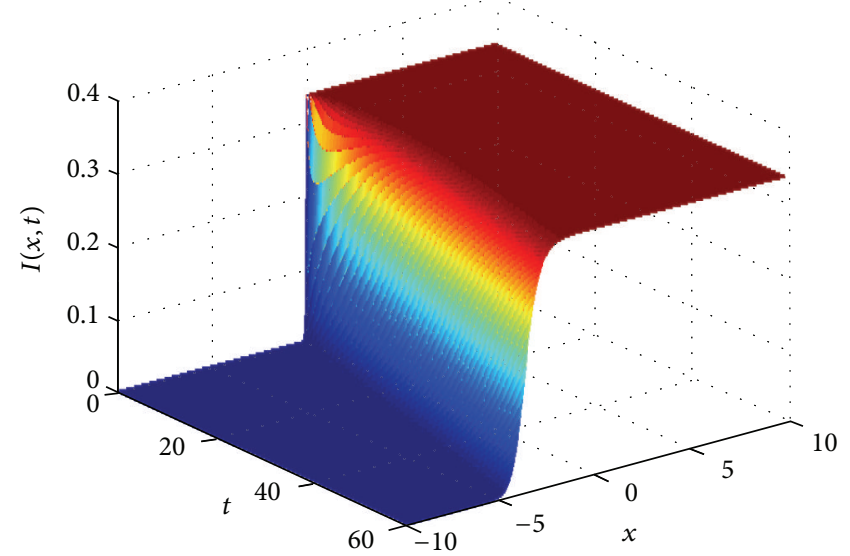

(b)

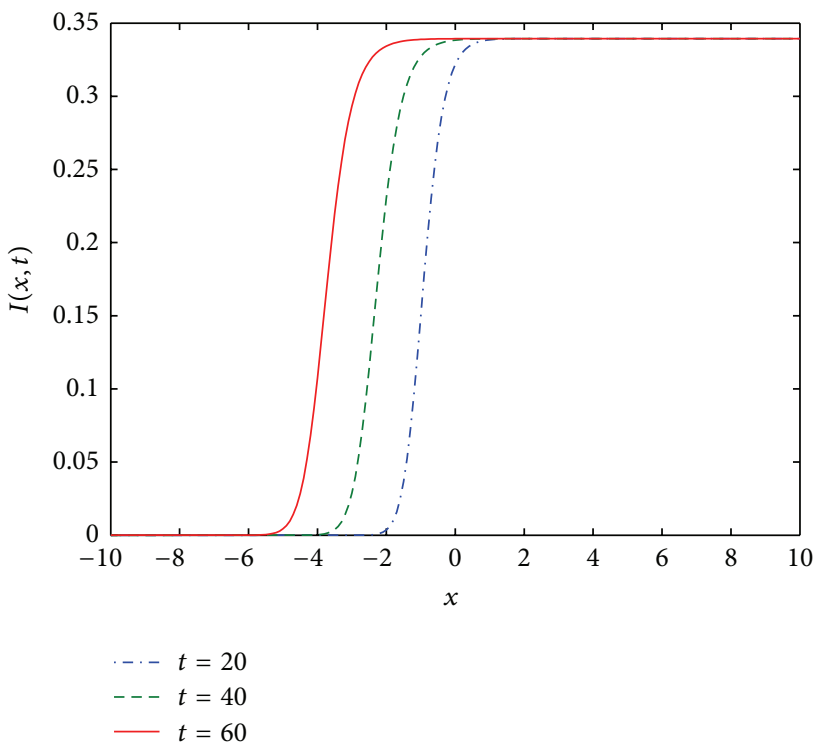

(d)

FIGURE 2: The traveling wave observed in system (5) with parameters: $\mu=0.1, \delta=\gamma=0.5, \beta=0.6, d_{1}=d_{2}=d_{3}=0.01$, and $\tau=0.75$.

and the humps in Figure 4(a) disappear in Figure 4(b), which indicates that the average latent period of the disease can impact not only the wave speed but also the monotonicity of the wave profile, as well as the level of the positive steady state. In biological meaning, large latent period of the disease could lead to low and slow infection, and may weaken the accumulative effect of infection.

Now we investigate the impact of transmission coefficient $\beta$. To this end, we fix $\delta=0.5, \tau=0.75, d_{1}=d_{2}=d_{3}=0.01$, and let $\beta$ vary. In this case, the values of $\mathscr{R}_{0}$ and $E^{*}$ are different (note that both $\mathscr{R}_{0}$ and $E^{*}$ are increasing in $\beta$ ). Figure 5 is the simulation results of (5). In Figure 5(a), $\beta=0.3$, but in Figure 5(b), we increase $\beta$ to 1.2. By contrasting the two figures, we can see that the travelling wave in Figure 5(b) is rather fast than that in Figure 5(a), but the travelling waves keep monotone as $\tau$ varies, which indicates that the trans- mission coefficient can obviously impact the wave speed, but it cannot change the monotonicity of the wave profile. In biological meaning, large transmission coefficient could lead to high and fast infection.

At last, we study the impact of an important parameter $\delta$, that is, the rate at which an individual in the recovered class reverts to the infective class. In the following, for convenience, we call $\delta$ the relapse rate. Now we fix $\beta=0.6, \tau=0.75$, and $d_{1}=d_{2}=d_{3}=0.01$, and let $\delta$ vary. In this case, the values of $\mathscr{R}_{0}$ and $E^{*}$ are also different (note that both $\mathscr{R}_{0}$ and $E^{*}$ are increasing in $\delta$ ). Figure 6 is the simulation results of (5). In Figure 6(a), $\delta=0.1$, but in Figure 6(b), we increase $\delta$ to 2. By contrasting the two figures, we can see that the travelling wave in Figure 6(b) is rather fast than that in Figure 6(a), but the travelling waves keep monotone as $\delta$ varies, which indicates that the relapse rate can obviously impact the wave speed, but 


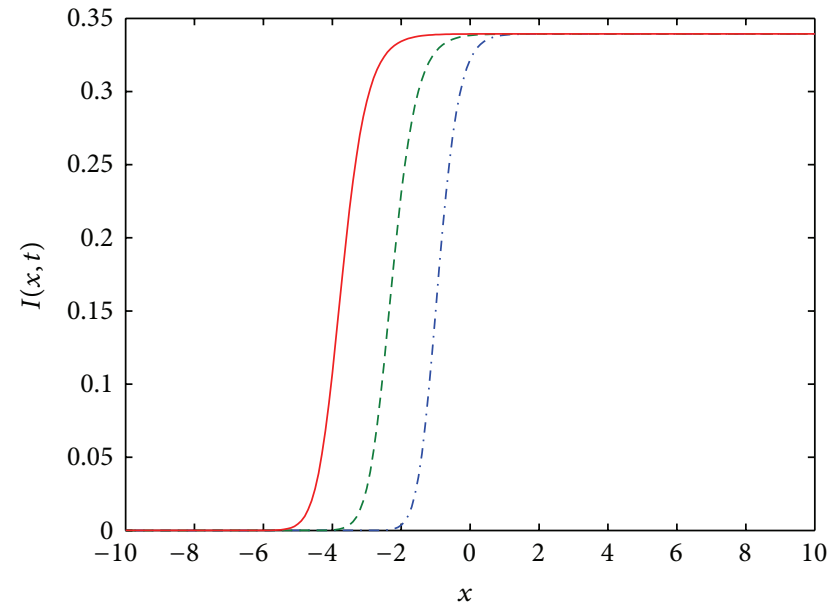

(a)

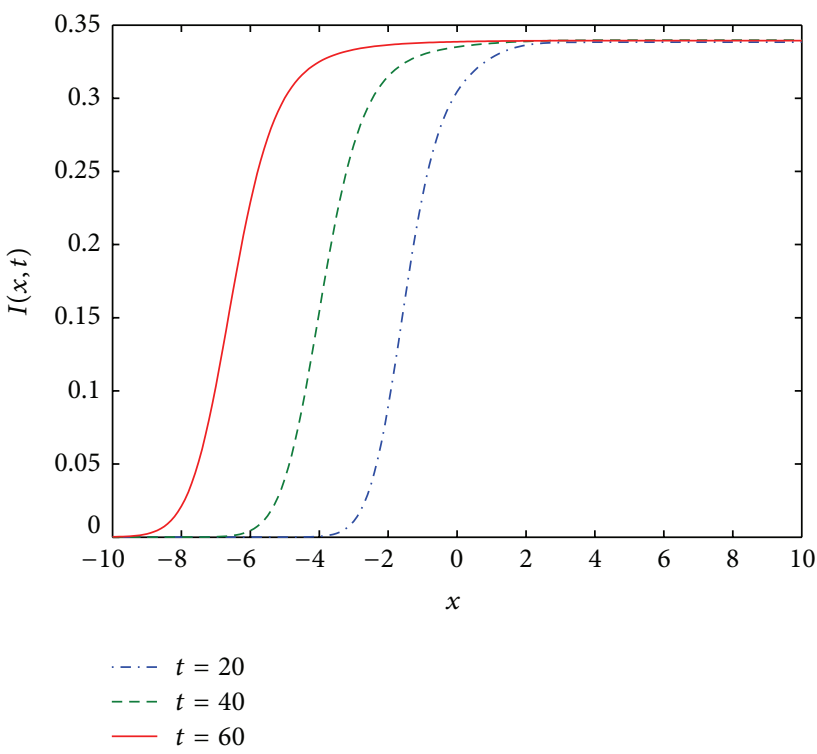

(c)

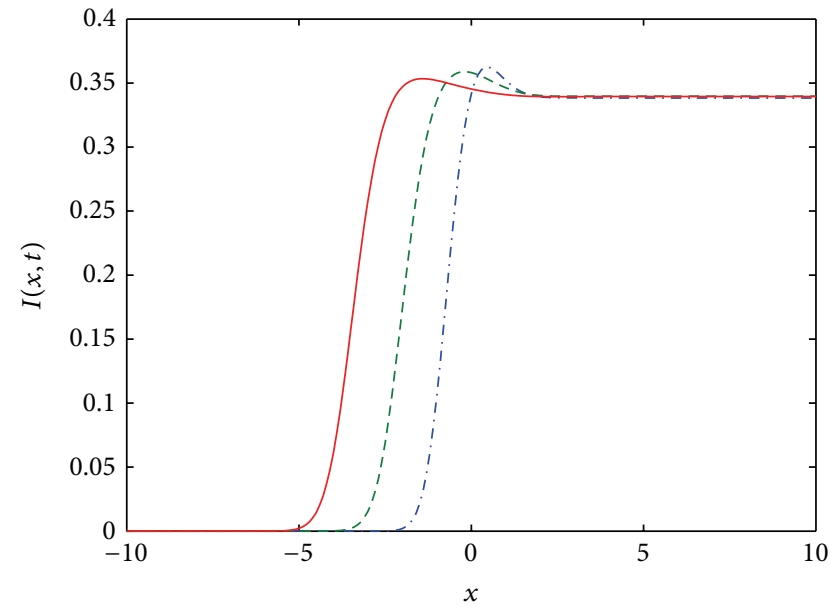

(b)

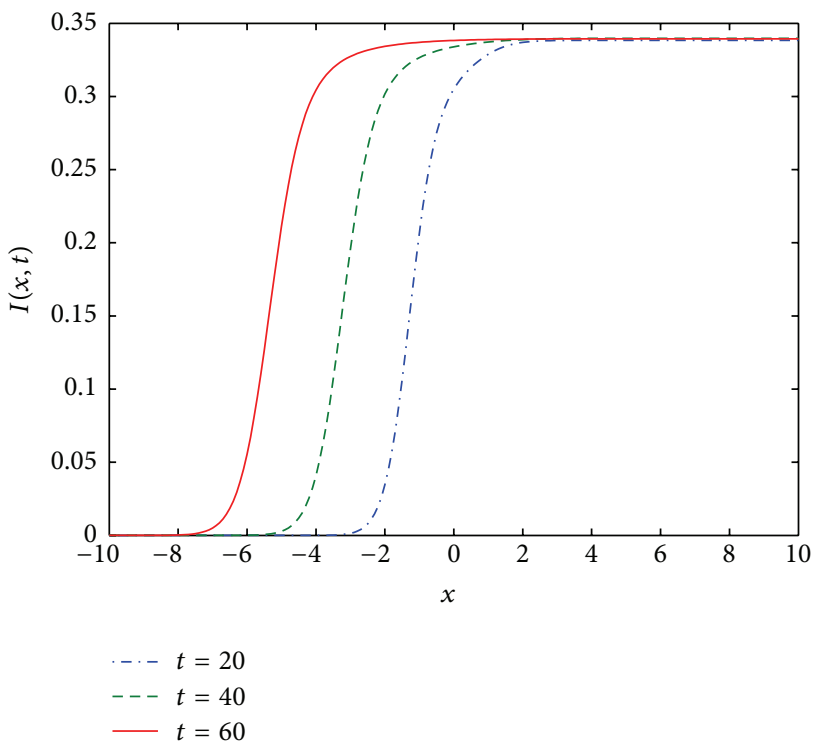

(d)

Figure 3: The traveling wave observed in system (5) with parameters: $\mu=0.1, \delta=\gamma=0.5, \beta=0.6, \tau=0.75$, and $d_{1}=d_{2}=d_{3}=0.01$ in (a); $d_{1}=0.04, d_{2}=d_{3}=0.01$ in (b); $d_{2}=0.04, d_{1}=d_{3}=0.01$ in (c); $d_{3}=0.04, d_{1}=d_{3}=0.01$ in (d).

it cannot change the monotonicity of the wave profile. In biological meaning, large transmission coefficient could lead to high and fast infection, which is similar to the case of transmission coefficient.

To end this section, we point out that for the parameters given above, the following properties of the spread speed $c$ can be obtained from the simulations and discussions above: $c$ is increasing in $d_{2}, d_{3}, \beta$, and $\delta$ but decreasing in $\tau$. In addition, small $\tau$ and large $d_{1}$ may lead to nonmonotone travelling waves.

\section{Conclusions}

In this paper, we formulated an SEIR model with latent period, relapse and spatial diffusion. The dynamics of problem
(5) and (6) was addressed. It was shown that the basic reproductive number of system (5) is given by $\mathscr{R}_{0}=\beta e^{-\mu \tau}(\mu+\delta) /$ $(\mu(\mu+\delta+\gamma))$, which describes the average number of newly infected individuals at the beginning of the infectious process. We have shown when the average number of newly infected individuals is less than unity, that is, $\mathscr{R}_{0}<1$; system (5) has a unique disease-free steady state $E_{0}(1,0,0)$, which is asymptotically stable; when the average number of newly infected individuals is greater than unity, that is, $\mathscr{R}_{0}>1$, system (5) admits two steady states, $E_{0}(1,0,0)$ and $E^{*}\left(S^{*}\right.$, $\left.I^{*}, R^{*}\right)$, where $S^{*}, I^{*}$, and $R^{*}$ are defined in (10). In this case, the endemic steady state $E^{*}$ is always stable, while the disease-free steady state $E_{0}$ is unstable. Clearly, the spatial diffusion cannot destabilize the spatially homogenous steady state. Then by using the technique of upper and lower 


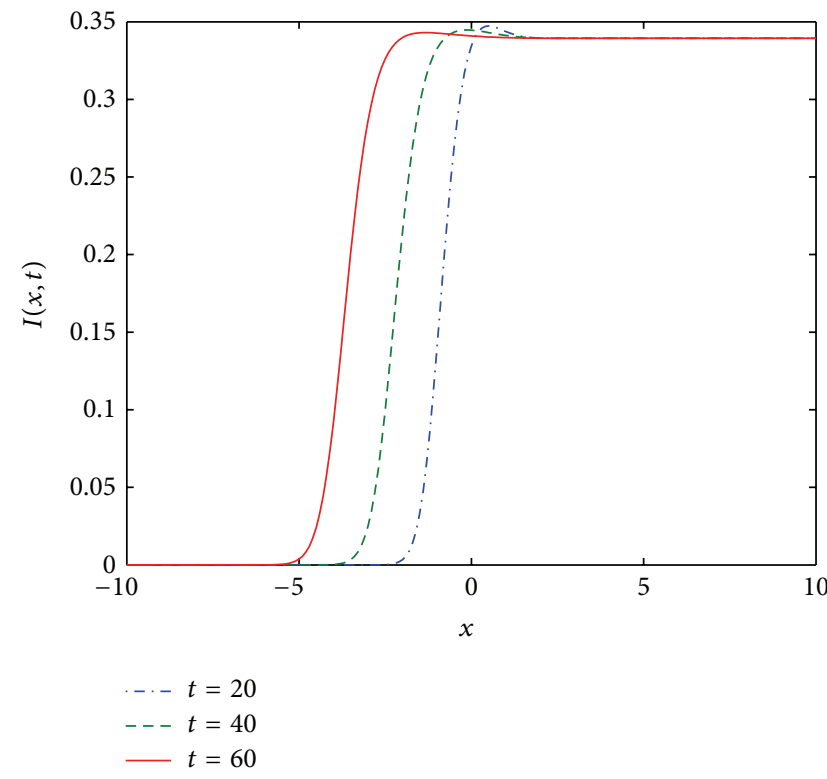

(a)

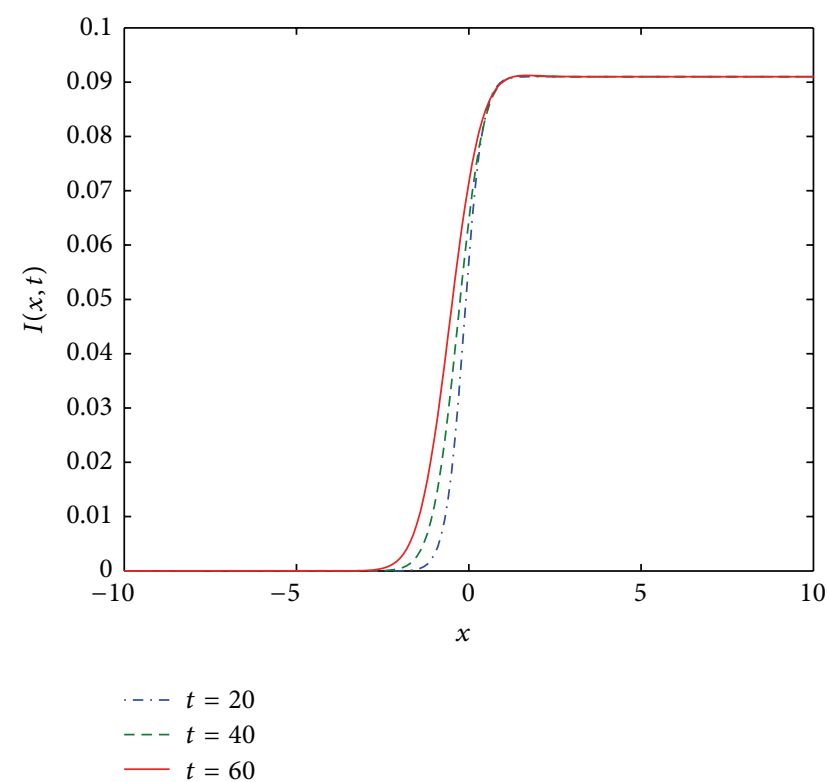

(b)

FIGURE 4: The traveling wave observed in system (5) with parameters: $\mu=0.1, \delta=\gamma=0.5, \beta=0.6, d_{1}=0.02, d_{2}=d_{3}=0.01$, and $\tau=0.75$ in (a); $\tau=7.5$ in (b).

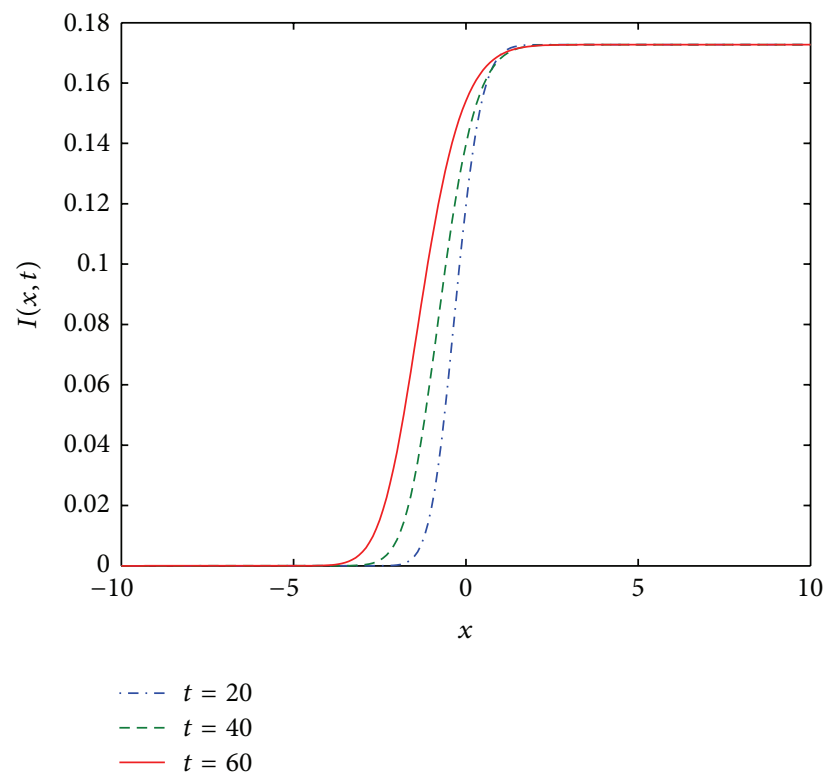

(a)

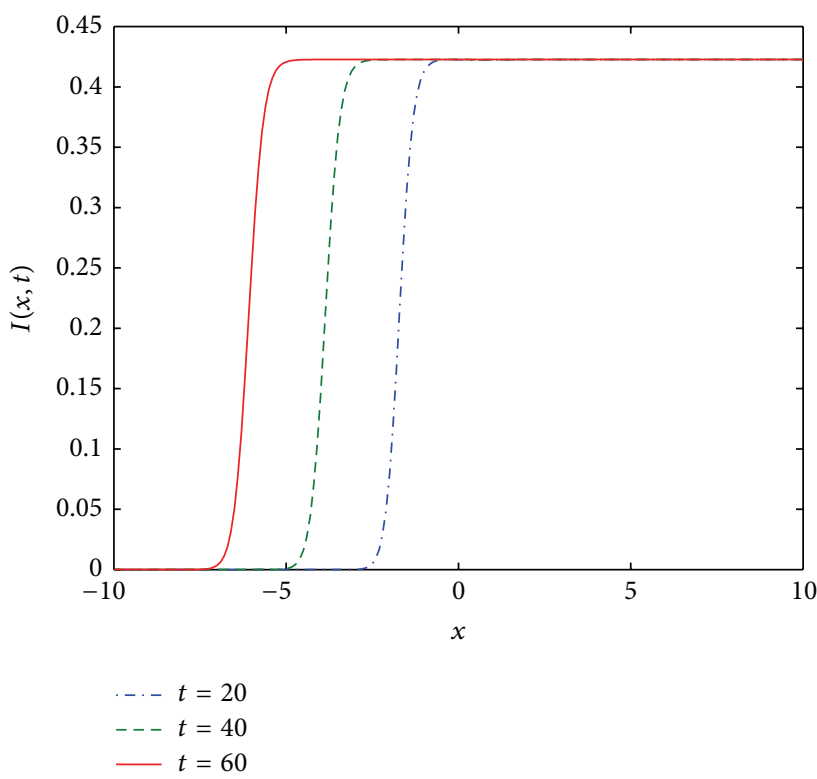

(b)

FIGURE 5: The traveling wave observed in system (5) with parameters: $\mu=0.1, \delta=\gamma=0.5, \tau=0.75, d_{1}=d_{2}=d_{3}=0.01$, and $\beta=0.3$ in (a); $\beta=1.2$ in (b).

solutions and Schauder's fixed point theorem, we derived the existence of a travelling wave solution connecting the disease-free steady state and the endemic steady state. Furthermore, we gave some numeric simulations to illustrate the main results, and combining with numeric simulations, we discussed the effects of some parameters on the dynamics of system (5).
From the discussion in Section 4, we could conclude that the travelling wave speed is increasing in the diffusion rates of infective and recovered individuals, the transmission coefficient, and the relapse rate, but decreasing in the average latent period of the disease. In addition, small latent period or large diffusion rate of susceptible individuals may lead to nonmonotone travelling waves. In other words, to slow down 


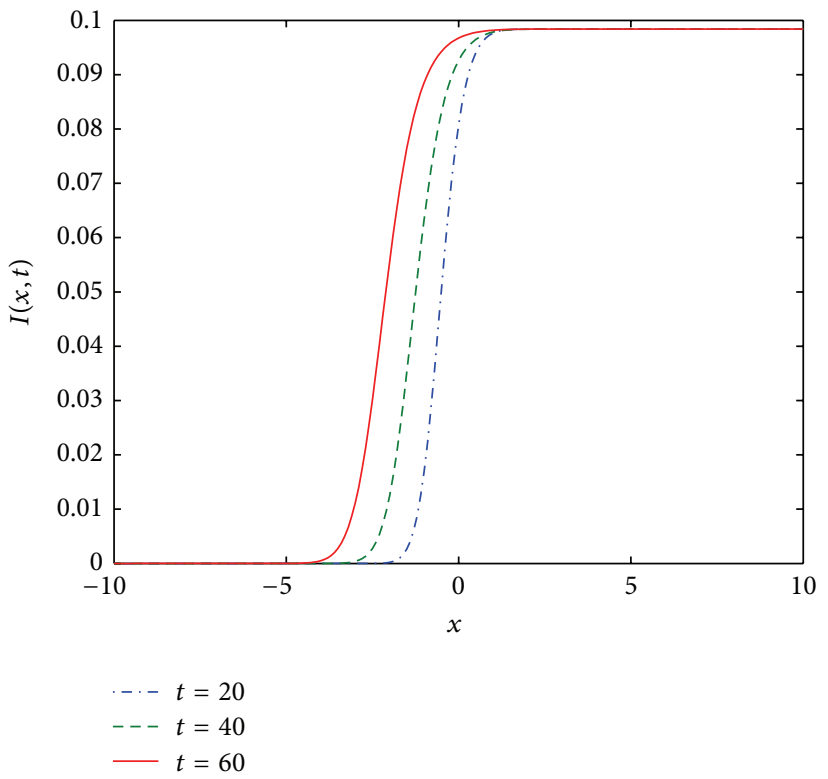

(a)

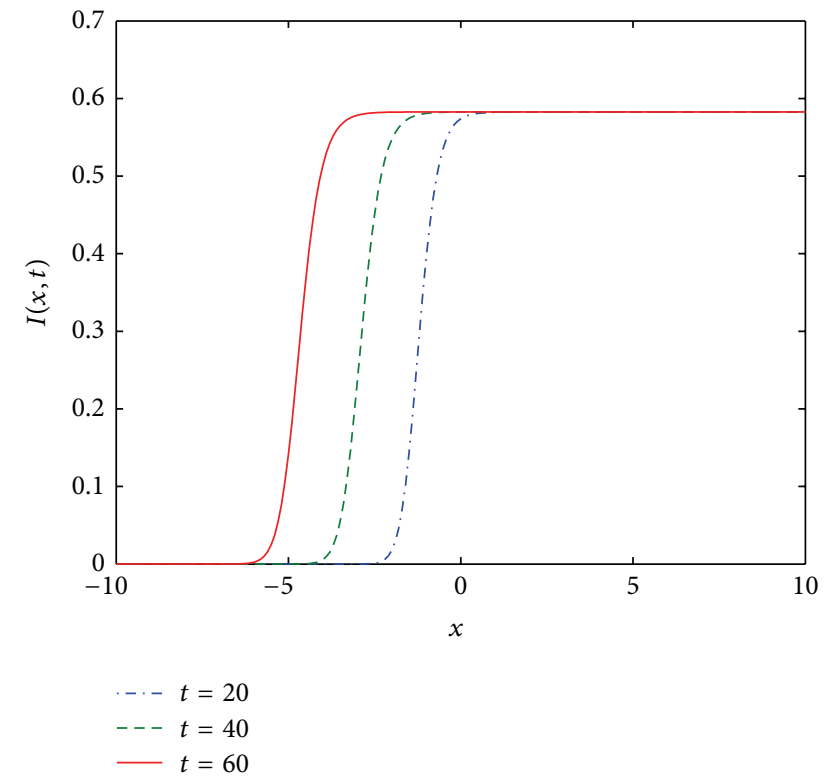

(b)

FiguRE 6: The traveling wave observed in system (5) with parameters: $\mu=0.1, \gamma=0.5, \beta=0.6, \tau=0.75, d_{1}=d_{2}=d_{3}=0.01$, and $\delta=0.1$ in (a); $\delta=2$ in (b).

or weaken the infection, we can reduce the diffusion rates, the transmission coefficient, or the relapse rate.

\section{Acknowledgments}

The authors are very grateful to the anonymous referees for careful reading and valuable comments which led to the improvement of their original paper. This work was supported by the National Natural Science Foundation of China (No. 11071254) and the Foundation of Shijiazhuang Mechanical Engineering College (Nos. JCB1201, YJJXM11004, and YJJXM12010).

\section{References}

[1] R. M. Anderson and W. Trewhella, "Population dynamics of the badger (Meles meles) and the epidemiology of bovine tuberculosis (Mycobacterium bovis)," Philosophical transactions of the Royal Society of London B, vol. 310, no. 1145, pp. 327-381, 1985.

[2] N. D. Barlow, "Non-linear transmission and simple models for bovine tuberculosis," Journal of Animal Ecology, vol. 69, no. 4, pp. 703-713, 2000.

[3] J. Chin, Control of Communicable Diseases Manual, American Public Health Association, Washington, DC, USA, 1999.

[4] S. W. Martin, Livestock Disease Eradication: Evaluation of the Cooperative State-Federal Bovine Tuberculosis Eradication Program, National Academy Press, Washington, DC, USA, 1994.

[5] K. E. VanLandingham, H. B. Marsteller, G. W. Ross, and F. G. Hayden, "Relapse of herpes simplex encephalitis after conventional acyclovir therapy," Journal of the American Medical Association, vol. 259, no. 7, pp. 1051-1053, 1988.
[6] P. van den Driessche, L. Wang, and X. Zou, "Modeling diseases with latency and relapse," Mathematical Biosciences and Engineering, vol. 4, no. 2, pp. 205-219, 2007.

[7] S. Liu, S. Wang, and L. Wang, "Global dynamics of delay epidemic models with nonlinear incidence rate and relapse," Nonlinear Analysis: Real World Applications, vol. 12, no. 1, pp. 119127, 2011.

[8] J. D. Murray, Mathematical Biology, Springer, New York, NY, USA, 3rd edition, 2002.

[9] J. V. Noble, "Geographic and temporal development of plagues," Nature, vol. 250, pp. 276-279, 1974.

[10] N. A. Maidana and H. M. Yang, "Spatial spreading of West Nile Virus described by traveling waves," Journal of Theoretical Bio$\log y$, vol. 258, no. 3, pp. 403-417, 2009.

[11] J. Huang and X. Zou, "Travelling wave solutions in delayed reaction diffusion systems with partial monotonicity," Acta Mathematicae Applicatae Sinica. English Series, vol. 22, no. 2, pp. 243256, 2006. 


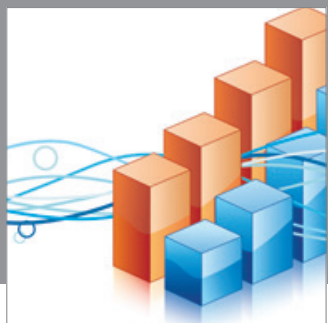

Advances in

Operations Research

mansans

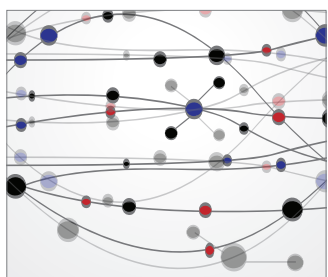

The Scientific World Journal
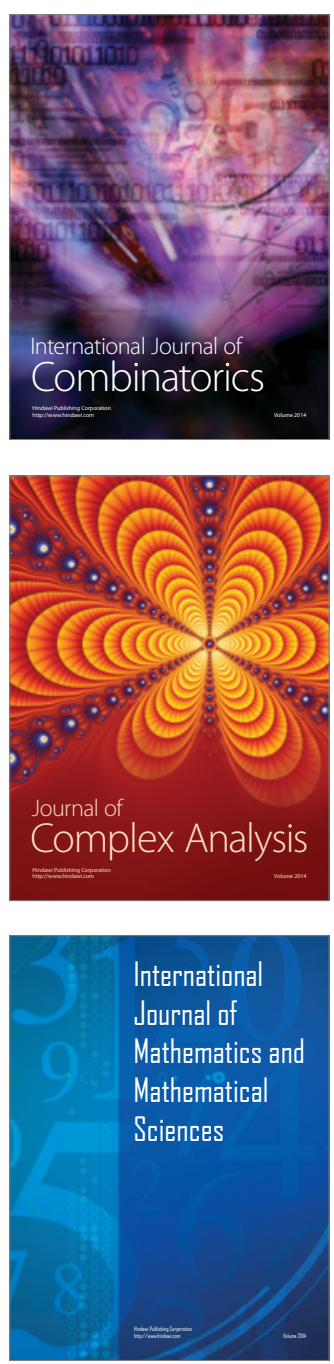
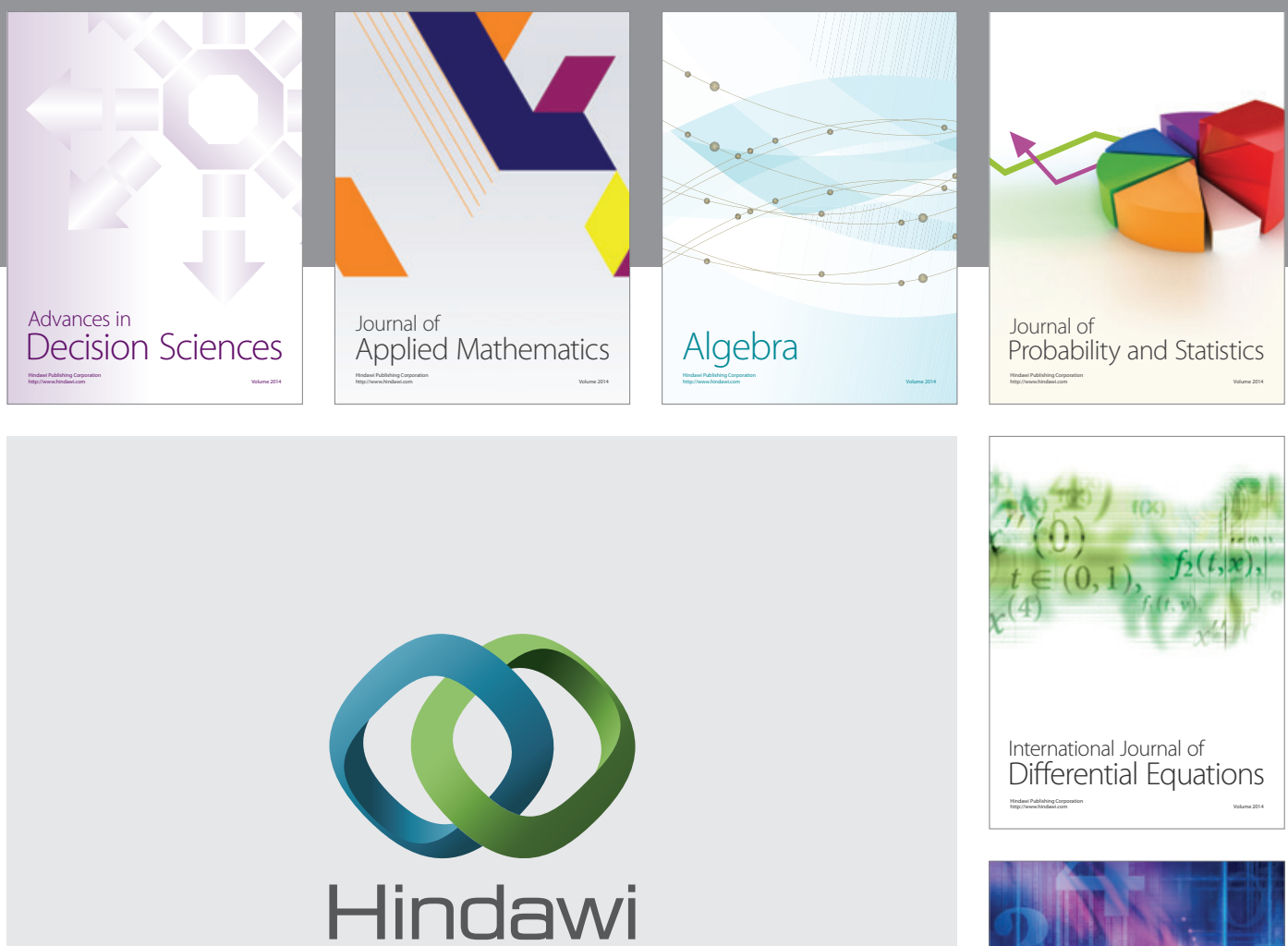

Submit your manuscripts at http://www.hindawi.com
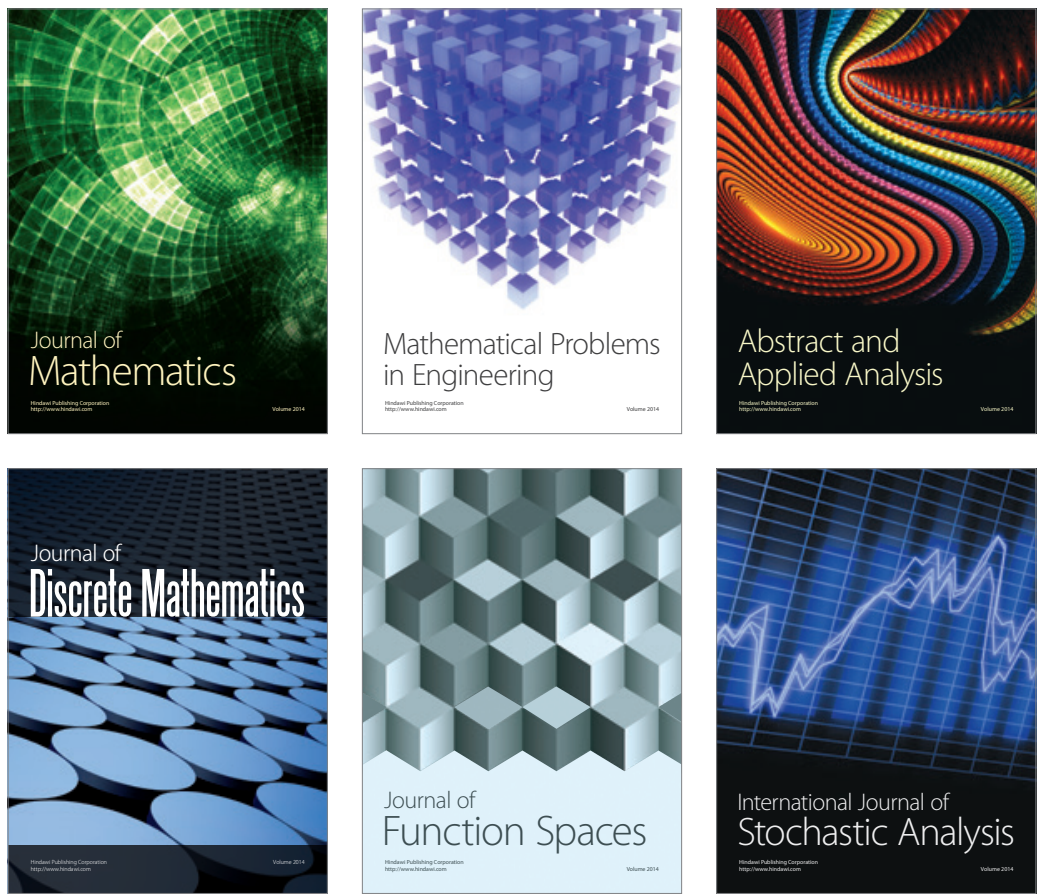

Journal of

Function Spaces

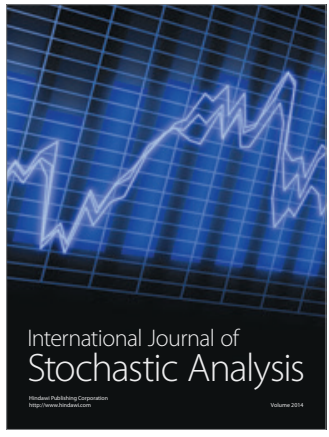

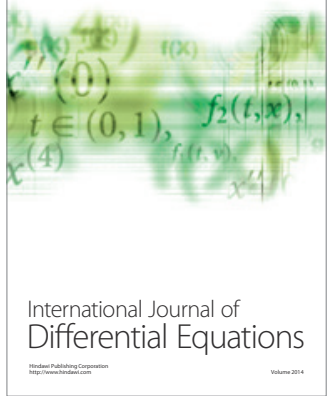
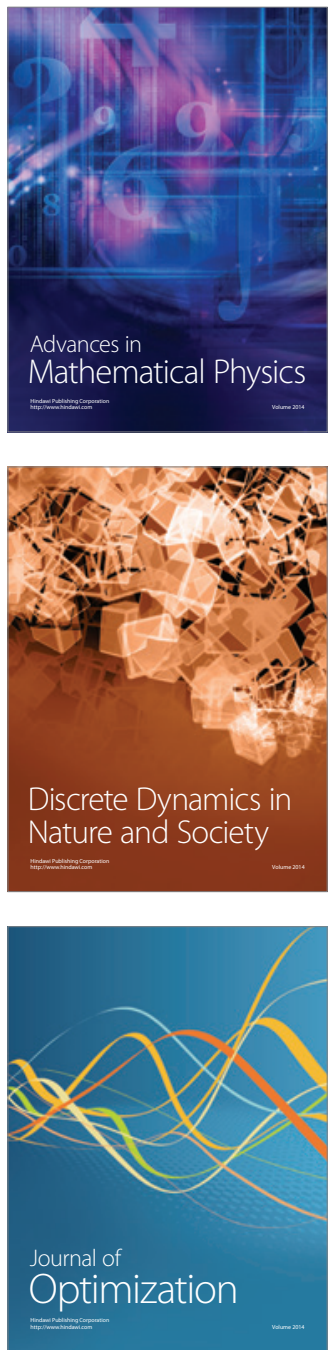